\title{
A Flexible Boundary Procedure for Hyperbolic Prob- lems: Multiple Penalty Terms Applied in a Domain
}

\author{
Jan Nordström ${ }^{1, *}$, Qaisar Abbas², Brittany A. Erickson ${ }^{3}$ and Hannes \\ Frenander ${ }^{4}$ \\ ${ }^{1}$ Department of Mathematics, Division of Computational Mathematics, Linköping \\ University, SE-581 83 Linköping, Sweden \\ 2 Department of Information Technology, Division of Scientific Computing, Uppsala \\ University, SE-751 05 Uppsala, Sweden \\ ${ }^{3}$ Department of Geological Sciences, San Diego State University, San Diego, CA \\ 92182-1020, USA \\ ${ }^{4}$ Department of Mathematics, Division of Computational Mathematics, Linköping \\ University, SE-581 83 Linköping, Sweden.
}

\begin{abstract}
A new weak boundary procedure for hyperbolic problems is presented. We consider high order finite difference operators of summation-by-parts form with weak boundary conditions and generalize that technique. The new boundary procedure is applied near boundaries in an extended domain where data is known. We show how to raise the order of accuracy of the scheme, how to modify the spectrum of the resulting operator and how to construct non-reflecting properties at the boundaries. The new boundary procedure is cheap, easy to implement and suitable for all numerical methods, not only finite difference methods, that employ weak boundary conditions. Numerical results that corroborate the analysis are presented.
\end{abstract}

AMS subject classifications: 35L05, 35L65, 35Q35, 65M06, 65M12 , 65Z05

Key words: Summation-by-parts, weak boundary conditions, penalty technique, high-order accuracy, finite difference schemes, stability, steady-state, non-reflecting boundary conditions.

\section{Introduction}

High order finite difference methods provide an efficient approach for problems in computational science. The efficiency can be used either to increase the accuracy for a fixed number of mesh points or to reduce the computational cost for a given accuracy by reducing the number of mesh points [27], [48]. The main drawback with high order finite

*Corresponding author. Email addresses: jan.nordstrom@liu.se (J. Nordström), qaisar.abbas@it.uu.se (Q. Abbas), berickson@projects.sdsu.edu (B. Erickson), hannes.frenander@liu.se (H. Frenander) 
difference methods is the complicated boundary treatment required to obtain a stable method.

Finite difference operators which satisfy the summation-by-parts (SBP) property [28, 29. 42], are central difference operators in the interior domain augmented with special stencils near the domain boundaries. These SBP operators in combination with weak well-posed boundary conditions lead to energy stability [6, 8, 16, 19, 31, 40, 41]. One such boundary treatment is the simultaneous approximation term (SAT) method [7], which linearly combines the partial differential equation to be solved with well-posed boundary conditions [5, 8, 34, 39].

In this paper we will extend this technique by applying the boundary conditions in an extended domain. As an introduction, consider the continuous one-dimensional right going $(a>0)$ advection problem

$$
u_{t}+a u_{x}=0, \quad 0 \leq x \leq 1, \quad t>0,
$$

with a boundary condition $u(0, t)=g_{0}(t)$ at $x=0$ for well-posedness. The energy method applied to (1.1) yields the following continuous energy rate

$$
\frac{d}{d t}\|u\|^{2}=a u(0, t)^{2}-a u(1, t)^{2},
$$

where $\|u\|^{2}=\int_{0}^{1} u^{2} d x$. By letting $u(0, t)=g_{0}(t)$, well-posedness follows.

Let the approximative solution at grid point $x_{i}$ be denoted $u_{i}$, and the discrete solution vector $\mathbf{u}^{T}=\left[u_{0}, u_{1}, \ldots, u_{N}\right]$. A finite difference approximation of (1.1) using an SBP operator with SAT treatment for the boundary condition can be written as

$$
\mathbf{u}_{t}+a P^{-1} Q \mathbf{u}=P^{-1} \alpha_{00}\left(u_{0}-g_{0}\right) e_{0},
$$

where the difference operator $P^{-1} Q$ approximates $d / d x, P$ is symmetric and positive definite, $Q+Q^{T}=B=\operatorname{diag}(-1,0, \ldots, 0,1), \alpha_{00}$ is called the penalty coefficient and $e_{0}=[1,0, \ldots, 0]^{T}$ is the unit vector that positions the penalty term at $i=0$. The discrete energy method on (1.3) gives

$$
\frac{d}{d t}\|\mathbf{u}\|_{P}^{2}=\left(a+2 \alpha_{00}\right) u_{0}^{2}-2 \alpha_{00} u_{0} g_{0}-a u_{N}^{2},
$$

where $\|\mathbf{u}\|_{P}^{2}=\mathbf{u}^{T} P \mathbf{u}$. Clearly, for $\alpha_{00} \leq-(a / 2)$, we have a bounded energy. Without imposing boundary conditions $\left(\alpha_{00}=0\right)$, the rate (1.4) mimics (1.2) perfectly. (A boundary condition at $x=0$ is necessary for stability, and it will be imposed below.) For more details using this technique, see [2,7, 8, 31, 42].

The SAT technique (weak imposition of boundary condition or penalty technique) is normally applied only at one grid point (as in the example above) [2, 3, 22, 23, 31, 36, 37, 44. 46]. However, in some cases, it is possible to impose the weak boundary conditions at multiple grid points. This has many advantages, and gives the designer of the scheme flexibility and many options. 
The flexibility mentioned above comes at a price. For accuracy reasons, the solution must be known at those grid points. Examples where one knows the boundary data in an extended domain include external fluid dynamics and various forms of wave propagation problems close to far-field boundaries. In some cases, the boundary data in the extended domain can be manufactured using Taylor's series expansions, repeated differentiation of the governing equations and given data at the boundary, see [20] for a description of that technique. The boundary data close to the boundaries can sometimes also be obtained due to simplifying circumstances such as special types of geometry or dependence of coordinate directions, see [17, 18, 47] for examples.

The boundary procedure that we present in this paper has many similarities to methods referred to as fringe region, sponge layers and buffer layers [4, 9, 21, 38] but is more general. It has also a clear connection to the Kållberg-Davies relaxation scheme often used in local weather prediction models, see [10, 24]. The data at the interface between the local and global domain can in this case be considered known from a global weather prediction model. Note that one only need know the data in a small domain close to the boundary or interface.

To illustrate the procedure, we add on additional penalty terms in (1.3) at two new grid points close to the boundary $x=0$ as

$$
\begin{aligned}
\mathbf{u}_{t}+a P^{-1} Q \mathbf{u}= & P^{-1}\left\{\alpha_{00}\left(u_{0}-g_{0}\right) e_{0}+\alpha_{01}\left(u_{1}-g_{1}\right) e_{0}\right. \\
& +\alpha_{10}\left(u_{0}-g_{0}\right) e_{1}+\alpha_{11}\left(u_{1}-g_{1}\right) e_{1}+\alpha_{12}\left(u_{2}-g_{2}\right) e_{1} \\
& \left.+\alpha_{21}\left(u_{1}-g_{1}\right) e_{2}+\alpha_{22}\left(u_{2}-g_{2}\right) e_{2}\right\},
\end{aligned}
$$

where $e_{1}=[0,1, \ldots, 0]^{T}$ and $e_{2}=[0,0,1, \ldots, 0]^{T} . \alpha_{i j}, i, j=0,1,2$ are the penalty coefficients and $g_{i}, i=0,1,2$ are the boundary data. Equation (1.5) is as accurate as the original scheme (1.3) as long as $g_{1}(\Delta x, t)$ and $g_{2}(2 \Delta x, t)$ are known. Let $g_{i}=0$ and use the energy method. The result corresponding to (1.4) with $g_{0}=0$ becomes

$$
\frac{d}{d t}\|\mathbf{u}\|_{P}^{2}=\left(\begin{array}{l}
u_{0} \\
u_{1} \\
u_{2}
\end{array}\right)^{T} \underbrace{\left(\begin{array}{ccc}
a+2 \alpha_{00} & \alpha_{01}+\alpha_{10} & 0 \\
\alpha_{01}+\alpha_{10} & 2 \alpha_{11} & \alpha_{12}+\alpha_{21} \\
0 & \alpha_{12}+\alpha_{21} & 2 \alpha_{22}
\end{array}\right)}_{R}\left(\begin{array}{l}
u_{0} \\
u_{1} \\
u_{2}
\end{array}\right)-a u_{N}^{2} .
$$

For stability of (1.6), we need to choose $\alpha_{i j}$, such that the matrix $R$ is negative semidefinite. (The most obvious choice would be $R_{i i} \leq 0$ and $R_{i j}=0$ for $i \neq j$.)

Once the stability requirements are fulfilled, a number of free parameters $\alpha_{i j}$ still exist. These can be used to

(i) increase the accuracy of the scheme,

(ii) modify the spectrum of the spatial operator,

(iii) change the wave speed of the error. 


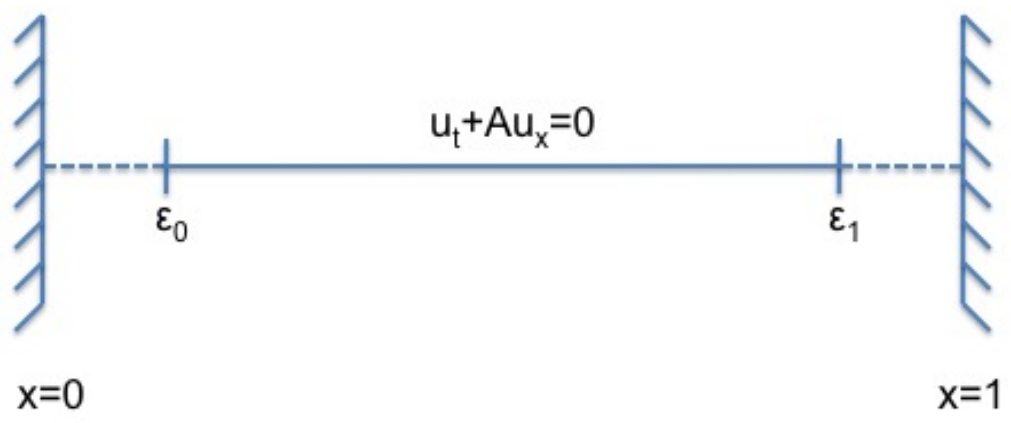

Figure 1: Illustration of multiple penalty domains $\left[0, \epsilon_{0}\right]$ and $\left[\epsilon_{1}, 1\right]$.

By using (i)-(iii) one can raise the order of accuracy of the scheme, increase the convergence rate to steady state and damp non-physical reflections at boundaries.

The rest of the paper proceeds as follows. In Section 2 we present a systematic procedure on how to generalize the multiple penalty technique to systems. Numerical experiments which illustrate the use of (i)-(iii) are presented in Section 3 for scalar problems and in Section 4 we consider two applications. The paper is concluded in Section 5.

\section{Multiple penalties for a hyperbolic system of equations}

The computational domain and the extended penalty regions in $\left[0, \epsilon_{0}\right]$ and $\left[\epsilon_{1}, 1\right]$ are shown in Figure 1. For a certain mesh, there are $p$ number of grid points in $\left[0, \epsilon_{0}\right]$ and $q$ number of grid points in $\left[\epsilon_{1}, 1\right]$. To demonstrate our new procedure we consider the symmetric hyperbolic problem

$$
\begin{aligned}
u_{t}+A u_{x} & =0, \quad x \in[0,1], \quad t>0, \\
A^{+} u(0, t) & =g_{L}(t), \\
A^{-} u(1, t) & =g_{R}(t),
\end{aligned}
$$

together with an initial condition which leads to a well-posed problem [19, 34, 35, 44, 45]. The $m \times m$ matrix $A$ can be split according to the sign of its eigenvalues as $A=A^{+}+A^{-}$, where $A^{+}=X \Lambda^{+} X^{T}$ and $A^{-}=X \Lambda^{-} X^{T}$. Here $\Lambda^{+}$and $\Lambda^{-}$contain the positive and negative (including zeros) eigenvalues respectively, and $X$ is the corresponding eigenvector matrix of $A$. The approximative solution at grid point $x_{i}$ is $\mathbf{u}_{i}=\left[\left(u_{0}\right)_{i},\left(u_{1}\right)_{i}, \ldots,\left(u_{m}\right)_{i}\right]$, for $i=0,1, \ldots, N$, and $\mathbf{u}=\left[\mathbf{u}_{0}, \mathbf{u}_{1}, \ldots, \mathbf{u}_{N}\right]^{T}$. We consider $\mathbf{u}_{\mathbf{e}}$ to be the known exact solution close to the boundaries and have a similar notation for the error $\mathbf{e}=\mathbf{u}_{\mathbf{e}}-\mathbf{u}$.

The Kronecker product $A \otimes B$ for matrices $A \in R^{m \times n}$ and $B \in R^{p \times q}$ is defined by

$$
A \otimes B=\left[\begin{array}{ccc}
a_{1,1} B & \ldots & a_{1, m} B \\
\vdots & \ddots & \vdots \\
a_{n, 1} B & \ldots & a_{m, n} B
\end{array}\right] .
$$


The Kronecker product is bilinear, associative and if the usual matrix products and matrix inverse are defined obeys

$$
(A \otimes B)(C \otimes D)=(A C \otimes B D), \quad(A \otimes B)^{-1, T}=A^{-1, T} \otimes B^{-1, T} .
$$

The semi-discrete form of (2.1) using SBP operators and a weak (SAT) treatment of boundary conditions can now be written

$$
\mathbf{u}_{t}+\left(P^{-1} Q \otimes A\right) \mathbf{u}=\left(P^{-1} \otimes I\right) R\left(\mathbf{u}-\mathbf{u}_{\mathbf{e}}\right),
$$

where $R$ is a general penalty matrix. By inserting the continuous solution $\mathbf{u}_{\mathbf{e}}$ (injected in the gridpoints) into (2.4) and subtracting (2.4) from the result we obtain the error equation

$$
\mathbf{e}_{t}+\left(P^{-1} Q \otimes A\right) \mathbf{e}=\left(P^{-1} \otimes I\right) R \mathbf{e}+T e,
$$

where Te is the truncation error. We split $R$ into $R=R_{0}+R_{m u l}$ where $R_{0}$ is the standard penalty term and $R_{m u l}$ an additional penalty term operating in the extended regions.

By multiplying (2.5) with $\mathbf{e}^{T}\left(P \otimes I_{m}\right)$ and adding its transpose, (we let $T e=0$ since this does not influence stability), we obtain

$$
\left.\frac{d}{d t}\|\mathbf{e}\|_{P}^{2}+\mathbf{e}^{T}\left[\left(Q+Q^{T}\right) \otimes A\right)\right] \mathbf{e}=\mathbf{e}^{T}\left[R_{0}+R_{0}^{T}+R_{m u l}+R_{m u l}^{T}\right] \mathbf{e} .
$$

To first determine the standard penalty term $R_{0}$ we let $R_{m u l}=0$, and choose

$$
R_{0}=\Sigma_{0} \otimes A^{+}+\Sigma_{N} \otimes A^{-}, \quad \Sigma_{0}=\left[\begin{array}{ccc}
\alpha_{00} & & \\
& \mathbf{0} & \\
& & \alpha_{N N}
\end{array}\right], \quad \Sigma_{N}=\left[\begin{array}{ccc}
\beta_{00} & & \\
& \mathbf{0} & \\
& & \beta_{N N}
\end{array}\right] .
$$

Since $P^{-1} Q$ is an SBP operator, it is straightforward to conclude that the energy rate 2.6 is bounded if

$$
\alpha_{00} \leq-\frac{1}{2} \quad \text { and } \quad \beta_{N N} \geq \frac{1}{2} \text {, while } \quad \alpha_{N N}=\beta_{00}=0 .
$$

The demands on $R_{m u l}$ will vary depending on the specific task we want it to accomplish.

\subsection{Increasing the accuracy of the scheme}

If the difference operator $P^{-1} Q$ is a uniformly high order difference operator with the same order of accuracy everywhere in the domain including the boundary region, then it is not a classical SBP operator. In this case we get additional terms in the symmetric part of $Q$,

$$
Q+Q^{T}=B+\tilde{B}_{s},
$$

where $\tilde{B}_{s}$ is a symmetric matrix with non-zero blocks of fixed sizes at the upper-left and lower-right corners. The size of the blocks depends on the order of the difference operator under consideration. 
Remark 2.1. There are many variants of $Q$, and they all depend on which choice of $P$ that we make. For stability reasons, see [32, 35, 43], we restrict ourselves to the standard diagonal SBP norm, which means that the accuracy at the boundary is half of that in the interior.

To increase the accuracy in a stable way, we add on a penalty matrix $R_{m u l}^{a c c}$ of the form

$$
R_{m u l}^{a c c}=\Sigma^{a c c} \otimes A, \quad \Sigma^{a c c}=\left[\begin{array}{ccc}
\Sigma_{L}^{a} & \mathbf{0} & \mathbf{0} \\
\mathbf{0} & \mathbf{0} & \mathbf{0} \\
\mathbf{0} & \mathbf{0} & \Sigma_{R}^{a}
\end{array}\right],
$$

where the energy rate (2.6) is bounded if $\mathbf{e}^{T}\left[R_{m u l}^{a c c}+R_{m u l}^{a c c T}\right] \mathbf{e}=\mathbf{e}^{T}\left(\tilde{\Sigma}^{a c c} \otimes A\right) \mathbf{e} \leq 0$ and $\tilde{\Sigma}^{a c c}=$ $-\tilde{B}_{s}+\Sigma^{a c c}+\Sigma^{a c c T}$. By choosing $\tilde{\Sigma}^{a c c}=0$ we get $\frac{d}{d t}\|\mathbf{e}\|_{P}^{2} \leq 0$ and energy stability.

Remark 2.2. For uniformly 2nd, 4 th and 6th order operators based on the standard diagonal SBP norm, weak boundary conditions must be imposed at one, four and six grid points respectively. The choice $P=\Delta x I$ ( $I$ is the identity matrix) for our uniformly high order operator, lowers the boundary data requirements. For 2nd, 4 th and 6th order operators, extra penalty terms will be required at one, two, and three grid points respectively. In Appendices $\mathrm{A}$ and $\mathrm{B}$ we present uniformly 2nd, 4th and 6th order accurate difference operators based on SBP and identity norms respectively.

\subsection{Modifying the spectrum of the spatial operator.}

A modified spectrum can increase the rate of convergence to steady-state. We start by applying multiple penalties on the incoming waves of the form

$$
R_{m u l}^{i n}=\Sigma_{L}^{i n} \otimes A^{+}+\Sigma_{R}^{i n} \otimes A^{-}, \quad \Sigma_{L}^{i n}=\left[\begin{array}{ccc}
\Sigma_{L}^{i} & \mathbf{0} & \mathbf{0} \\
\mathbf{0} & \mathbf{0} & \mathbf{0} \\
\mathbf{0} & \mathbf{0} & \mathbf{0}
\end{array}\right], \quad \Sigma_{R}^{i n}=\left[\begin{array}{ccc}
\mathbf{0} & \mathbf{0} & \mathbf{0} \\
\mathbf{0} & \mathbf{0} & \mathbf{0} \\
\mathbf{0} & \mathbf{0} & \Sigma_{R}^{i}
\end{array}\right] .
$$

The penalty matrix $\Sigma_{L}^{i}$ covers the domain $\left[0, \epsilon_{0}\right]$ and $\Sigma_{R}^{i}$ covers the domain $\left[\epsilon_{1}, 1\right]$. They are of dimension $p$ and $q$ respectively. The energy rate (2.6) is bounded if

$$
\mathbf{e}^{T}\left[R_{m u l}^{i n}+R_{m u l}^{i n T}\right] \mathbf{e}=\mathbf{e}_{p}^{T}\left(\Sigma_{L}^{i}+\Sigma_{L}^{i T} \otimes A^{+}\right) \mathbf{e}_{p}+\mathbf{e}_{q}^{T}\left(\Sigma_{R}^{i}+\Sigma_{R}^{i T} \otimes A^{-}\right) \mathbf{e}_{q} \leq 0 .
$$

Here $\mathbf{e}_{p}$ is the error vector in $\left[0, \epsilon_{0}\right]$, and $\mathbf{e}_{q}$ is the error vector in $\left[\epsilon_{1}, 1\right]$.

One can also modify waves passing out of the domain by choosing

$$
R_{m u l}^{\text {out }}=\Sigma_{L}^{\text {out }} \otimes A^{-}+\Sigma_{R}^{o u t} \otimes A^{+}, \quad \Sigma_{L}^{\text {out }}=\left[\begin{array}{ccc}
\Sigma_{L}^{o} & \mathbf{0} & \mathbf{0} \\
\mathbf{0} & \mathbf{0} & \mathbf{0} \\
\mathbf{0} & \mathbf{0} & \mathbf{0}
\end{array}\right], \quad \Sigma_{R}^{\text {out }}=\left[\begin{array}{ccc}
\mathbf{0} & \mathbf{0} & \mathbf{0} \\
\mathbf{0} & \mathbf{0} & \mathbf{0} \\
\mathbf{0} & \mathbf{0} & \Sigma_{R}^{o}
\end{array}\right] .
$$


In (2.12), $\Sigma_{L, R}^{\text {out }}$ adds damping on the outgoing waves at the left and right boundaries respectively. The energy rate (2.6) is bounded if

$$
\mathbf{e}^{T}\left[R_{m u l}^{o u t}+R_{m u l}^{o u t T}\right] \mathbf{e}=\mathbf{e}_{p}^{T}\left(\Sigma_{L}^{o}+\Sigma_{L}^{o T} \otimes A^{-}\right) \mathbf{e}_{p}+\mathbf{e}_{q}^{T}\left(\Sigma_{R}^{o}+\Sigma_{R}^{o T} \otimes A^{+}\right) \mathbf{e}_{q} \leq 0 .
$$

The choices of penalties made above move the spectrum to the right in the complex plane as will be shown below.

\subsection{Changing the wave speed}

For the construction of boundary closures which change the error propagation, we use

$$
R_{\text {mul }}^{\text {wave }}=\Sigma_{+}^{\text {wave }} \otimes A^{+}+\Sigma_{-}^{\text {wave }} \otimes A^{-} .
$$

We partition $Q$ and $\Sigma_{+,-}^{\text {wave }}$ as

$Q=\left[\begin{array}{ccc}Q_{L} & \mathbf{0} & \mathbf{0} \\ \mathbf{0} & Q_{M} & \mathbf{0} \\ \mathbf{0} & \mathbf{0} & Q_{R}\end{array}\right], \quad \Sigma_{+}^{\text {wave }}=\left[\begin{array}{ccc}\alpha_{L}^{+} Q_{L} & \mathbf{0} & \mathbf{0} \\ \mathbf{0} & \mathbf{0} & \mathbf{0} \\ \mathbf{0} & \mathbf{0} & \alpha_{R}^{+} Q_{R}\end{array}\right], \quad \Sigma_{-}^{\text {wave }}=\left[\begin{array}{ccc}\alpha_{L}^{-} Q_{L} & \mathbf{0} & \mathbf{0} \\ \mathbf{0} & \mathbf{0} & \mathbf{0} \\ \mathbf{0} & \mathbf{0} & \alpha_{R}^{-} Q_{R}\end{array}\right]$.

Here $Q_{L}$ and $Q_{R}$ are square matrices of size $p$ and $q$ respectively and $\alpha_{L}, \alpha_{R}$ are scalars. The relation (2.5) is modified to

$$
\mathbf{e}_{t}+\left(P^{-1} \tilde{Q}_{+} \otimes A^{+}\right) \mathbf{e}+\left(P^{-1} \tilde{Q}_{-} \otimes A^{-}\right) \mathbf{e}=\left(P^{-1} \otimes I\right) R_{0} \mathbf{e},
$$

where $\tilde{Q}_{+,-}=Q-\Sigma_{+,-}^{\text {wave }}$ is given by

$$
\tilde{Q}_{+,-}=\left[\begin{array}{ccc}
\left(1-\alpha_{L}^{+,-}\right) Q_{L} & \mathbf{0} & \mathbf{0} \\
\mathbf{0} & Q_{M} & \mathbf{0} \\
\mathbf{0} & \mathbf{0} & \left(1-\alpha_{R}^{+,-}\right) Q_{R}
\end{array}\right]
$$

By choosing $\alpha_{L}^{+,-}$and $\alpha_{R}^{+,-}$properly, we can control the wave speed of the errors in the extended regions.

The energy rate (2.6) in view of (2.14) becomes

$$
\frac{d}{d t}\|\mathbf{e}\|_{P}^{2}=\tilde{\alpha}_{00} \mathbf{e}_{0}^{T} A^{+} \mathbf{e}_{0}+\tilde{\alpha}_{N N} \mathbf{e}_{N}^{T} A^{+} \mathbf{e}_{N}+\tilde{\beta}_{00} \mathbf{e}_{0}^{T} A^{-} \mathbf{e}_{0}+\tilde{\beta}_{N N} \mathbf{e}_{N}^{T} A^{-} \mathbf{e}_{N},
$$

where $\mathbf{e}_{0}$ and $\mathbf{e}_{N}$ are the elements of the error vector $\mathbf{e}$ (not the unit vectors at grid points $0, N)$. For stability we require

$$
\begin{aligned}
& \tilde{\alpha}_{00}=\left(1-\alpha_{L}^{+}\right)+2 \alpha_{00} \leq 0, \quad \tilde{\alpha}_{N N}=-\left(1-\alpha_{R}^{+}\right)+2 \alpha_{N N} \leq 0, \\
& \tilde{\beta}_{00}=\left(1-\alpha_{L}^{-}\right)+2 \beta_{00} \geq 0, \quad \tilde{\beta}_{N N}=-\left(1-\alpha_{R}^{-}\right)+2 \beta_{N N} \geq 0 .
\end{aligned}
$$




\subsection{Other possible combinations of penalty terms}

We noted above that $R_{0}$ in (2.7) is sufficient to make the SBP scheme stable. All other additions of $R_{m u l}\left(R_{m u l}^{a c c}, R_{m u l}^{i n}, R_{m u l}^{o u t}, R_{m u l}^{\text {wave }}\right)$ are specifically constructed for the tasks we want them to perform. It is of course possible to combine these additional matrices. For effects on the convergence rate to steady-state, we can use (2.11) and (2.12) as

$$
R_{m u l}=R_{m u l}^{i n}+R_{m u l}^{o u t} .
$$

Another useful combination of (2.11), (2.12) and (2.13) is given by

$$
R_{m u l}=R_{m u l}^{\text {wave }}+R_{m u l}^{i n}+R_{m u l}^{o u t} .
$$

By using (2.17) we can control the wave speed of errors in the penalty regions and damp the reflections at the same time. If $P^{-1} Q$ is a uniformly high order operator instead of an SBP operator, we can use (2.10) for stability, while adding any one of the combinations (2.16) and 2.17).

\section{Numerical experiments}

Here we illustrate and evaluate the validity and usefulness of the preceding theory.

\subsection{Higher order accuracy}

We consider the problems (1.1) and (2.1) and the corresponding semi-discrete formulation (2.4), 2.9) and penalty matrix (2.10). We take $P^{-1} Q$ as a uniformly fourth and sixth order accurate difference operator based on the diagonal SBP norm as defined in Appendix A. The results compared with the standard SBP schemes in the scalar case are shown in Tables 1 and 2 while the result for the system case with $A=X \Lambda X^{T}$ and

$$
A=\left[\begin{array}{ll}
0 & 1 \\
1 & 0
\end{array}\right], \quad \Lambda=\left[\begin{array}{cc}
1 & 0 \\
0 & -1
\end{array}\right], \quad X=\frac{1}{\sqrt{2}}\left[\begin{array}{cc}
1 & 1 \\
1 & -1
\end{array}\right]
$$

is shown in Table 3 . The results clearly corroborate the theory.

Remark 3.1. Uniformly fourth and sixth order difference operators based on the identity norm given in Appendix B were also shown to have the same accuracy.

Remark 3.2. In some cases, the boundary data in the extended regions can be manufactured using Taylor's series expansions, repeated differentiation of the governing equations and given data at the boundary, see [20] and Appendix $\mathrm{C}$ where this technique is exemplified. 
Table 1: $L_{2}$-error and convergence rates $(q)$, for 3 rd order SBP and uniformly 4 th order schemes in the scalar case.

\begin{tabular}{cccccc}
\hline Points & \multicolumn{2}{c}{ 3rd order SBP scheme } & & \multicolumn{2}{c}{ uniformly 4 th order scheme } \\
\cline { 2 - 3 } \cline { 5 - 5 } & $l_{2}$-error & $\mathrm{q}$ & & $l_{2}$-error & $\mathrm{q}$ \\
\hline 21 & $7.19 e-03$ & - & & $7.44 e-04$ & - \\
41 & $9.16 e-04$ & 2.97 & & $5.00 e-05$ & 3.89 \\
81 & $1.17 e-04$ & 2.97 & & $3.23 e-06$ & 3.95 \\
161 & $1.48 e-05$ & 2.98 & & $2.05 e-07$ & 3.98 \\
321 & $1.87 e-06$ & 2.99 & & $1.29 e-08$ & 3.99 \\
\hline
\end{tabular}

Table 2: $L_{2}$-error and convergence rates $(q)$, for 4 th order SBP and uniformly 6 th order schemes in the scalar case.

\begin{tabular}{cccccc}
\hline Points & \multicolumn{2}{c}{ 4th order SBP scheme } & & \multicolumn{2}{c}{ uniformly 6th order scheme } \\
\cline { 2 - 3 } \cline { 5 - 5 } & $l_{2}$-error & $\mathrm{q}$ & & $l_{2}$-error & $\mathrm{q}$ \\
\hline 21 & $8.11 e-03$ & - & & $7.18 e-06$ & - \\
41 & $7.61 e-04$ & 3.41 & & $1.96 e-07$ & 5.19 \\
81 & $5.29 e-05$ & 3.84 & & $3.76 e-09$ & 5.70 \\
161 & $3.41 e-06$ & 3.95 & & $6.41 e-11$ & 5.87 \\
321 & $2.16 e-07$ & 3.98 & & $1.04 e-12$ & 5.94 \\
\hline
\end{tabular}

Table 3: $L_{2}$-error and convergence rates $(q)$, for uniformly 4 th and 6 th order schemes in the system case.

\begin{tabular}{|c|c|c|c|c|}
\hline \multirow[t]{2}{*}{ Points } & \multicolumn{2}{|c|}{ uniformly 4th order scheme } & \multicolumn{2}{|c|}{ uniformly 6th order scheme } \\
\hline & $l_{2}$-error & $q$ & $l_{2}$-error & $q$ \\
\hline 21 & $4.10 e-04$ & - & $6.60 e-06$ & - \\
\hline 41 & $2.64 e-05$ & 3.95 & $1.18 e-07$ & 5.80 \\
\hline 81 & $1.66 e-06$ & 3.99 & $2.02 e-09$ & 5.87 \\
\hline 161 & $1.03 e-07$ & 4.00 & $3.29 e-13$ & 5.93 \\
\hline 321 & $6.48 e-09$ & 4.00 & $5.26 e-13$ & 5.96 \\
\hline
\end{tabular}




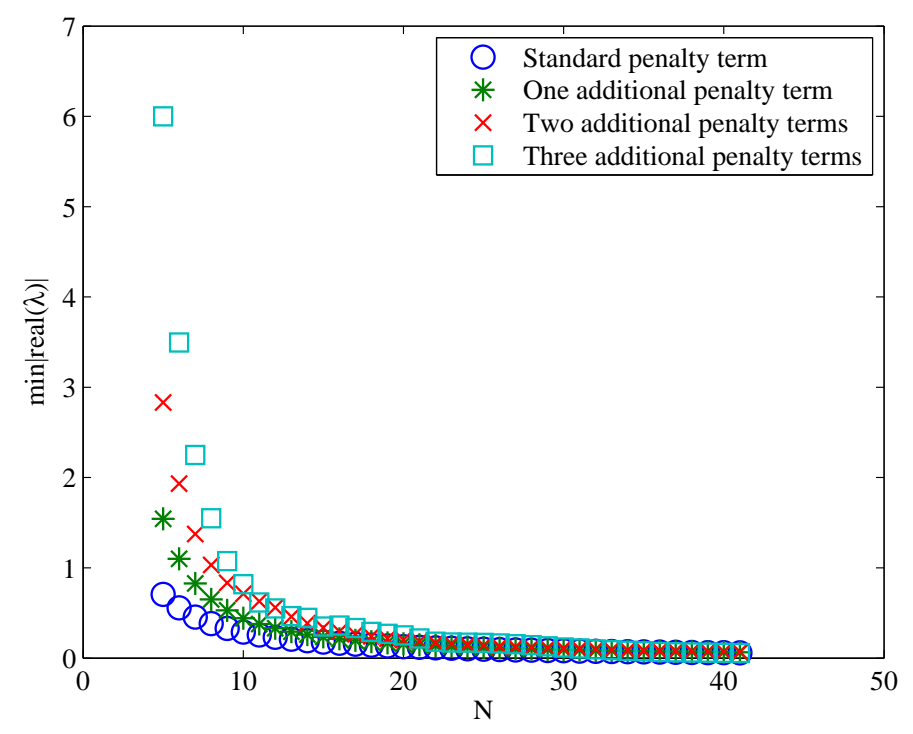

Figure 2: The effect of adding penalty terms on more grid points to the spectrum. We show $\min |\operatorname{real}(\lambda)|$ for various $N$.

\subsection{Steady-state computations}

Consider the advection problem (1.1) with initial data $u(x, 0)=1+e^{-100(x-0.5)^{2}}$ and boundary data $g_{0}=1$. Equations (2.5), 2.7) and 2.11) lead to (let $\mathrm{Te}=0$ )

$$
\mathbf{e}_{t}+a P^{-1} Q \mathbf{e}=a P^{-1}\left(\Sigma_{0}+\Sigma_{L}^{i n}\right) \mathbf{e} .
$$

We choose the penalty matrices $\Sigma_{0}$ and $\Sigma_{L}^{\text {in }}$ in (3.1) in such a way that eigenvalues shift away from the imaginary axis, see Figure 2. The largest effect is obtained for coarse meshes.

In the calculations below we plot the $l_{2}$-norm of $\mathbf{e}=\mathbf{u}-\mathbf{u}_{\mathbf{e}}$ as a function of time. In Figure 3 for $N=11$ we see the effect of adding penalties on more grid points. It results in an increased convergence rate to steady-state. The convergence rate decreases for a fixed number of multiple penalties as can be seen by comparing Figure 4 for $N=21$ with Figure 3 for $N=11$. It can also be seen that the convergence rate remains approximately constant if one adds on penalty terms as the mesh is refined, see Figure 5.

Next we consider (2.5), 2.7) and (2.16), so that (3.1) is modified to

$$
\mathbf{e}_{t}+a P^{-1} Q \mathbf{e}=a P^{-1}\left(\Sigma_{0}+\Sigma_{L}^{i n}+\Sigma_{R}^{o u t}\right) \mathbf{e} .
$$

The last additional term in (3.2) damps the outgoing waves close to the outflow boundary. There are many ways to choose the elements of the matrix $\Sigma_{R}^{o u t}$ in (3.2). For simplicity we consider a constant diagonal matrix of the form $\Sigma_{R}^{o u t}=c_{0} \operatorname{diag}(1, \ldots, 1)$ in $\left[\epsilon_{1}, 1\right]$, where 


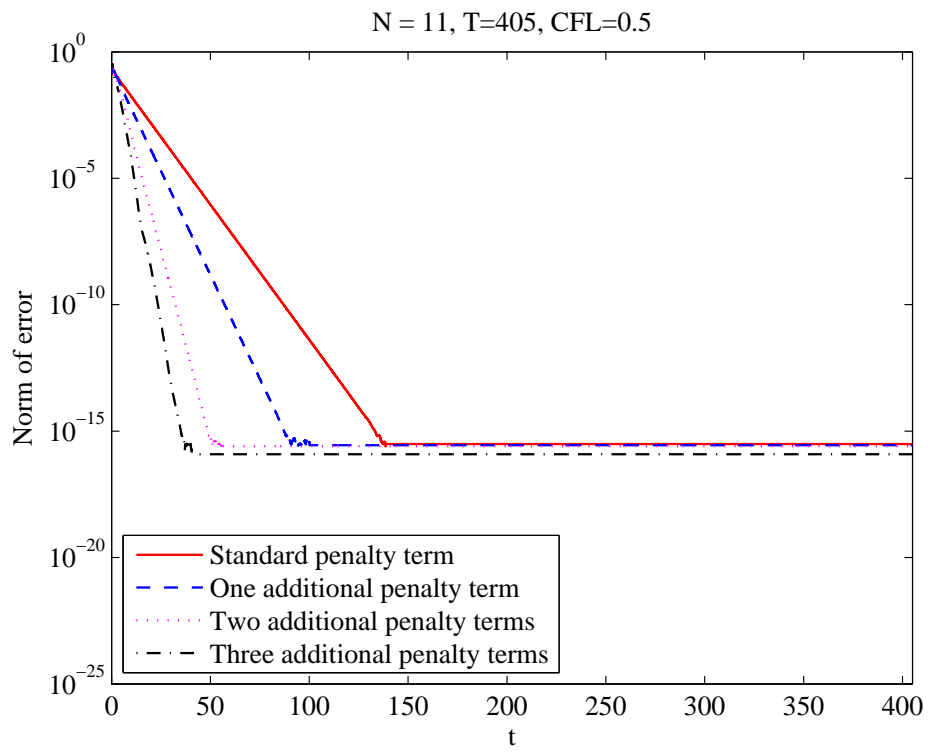

Figure 3: The effect of adding penalty terms on more grid points and corresponding convergence rates to steady-states for $N=11$.

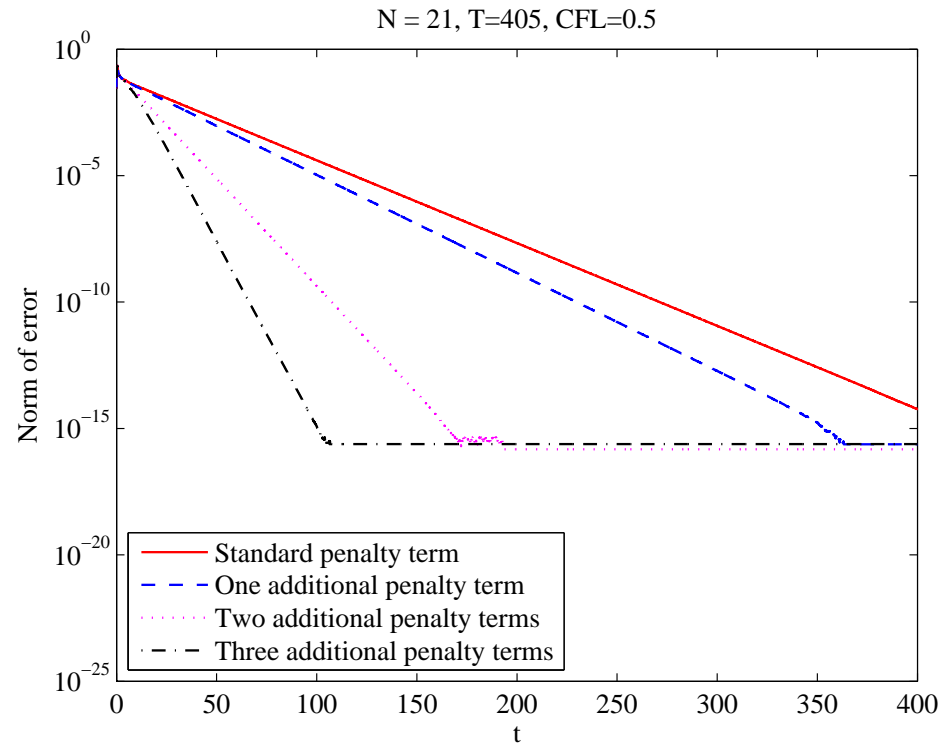

Figure 4: The effect of adding penalty terms on more grid points and corresponding convergence rates to steady-states for $N=21$. 


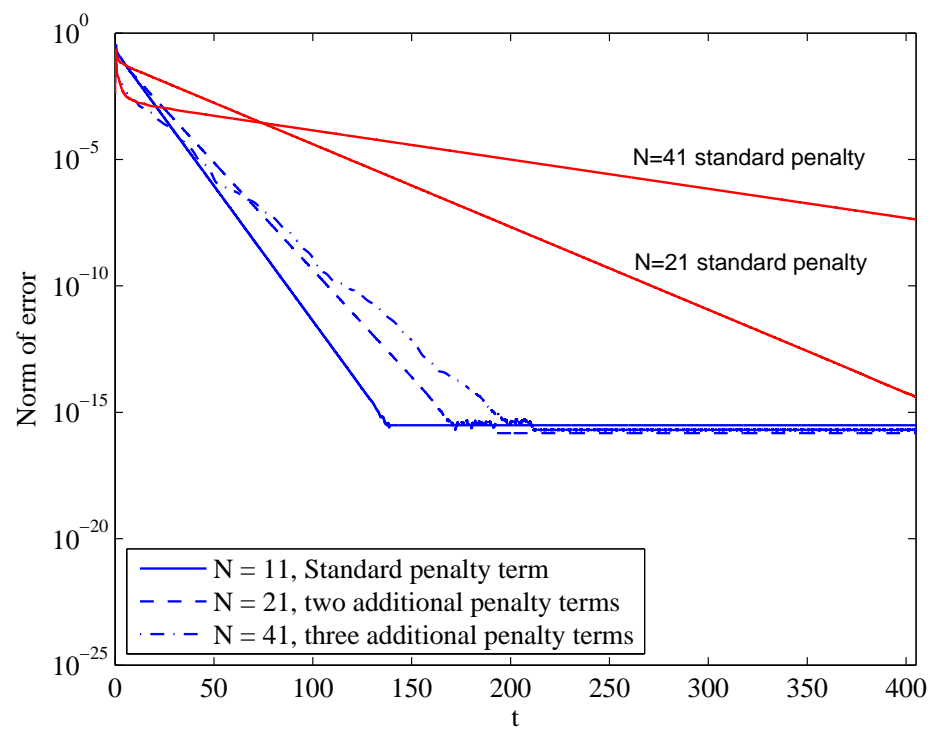

Figure 5: The effect of adding multiple penalty on mesh refinement.

$c_{0}$ is a tuning parameter. The convergence rates for $N=11$ are compared with the corresponding results using the standard penalty and one additional penalty as shown in Figure 6. As can be seen, the convergence rate to steady-state is improved considerably.

\subsection{Changing wave speeds}

We consider the formulation (2.14) applied to the scalar problem 1.1

$$
\mathbf{e}_{t}+a P^{-1} \tilde{Q} \mathbf{e}=a P^{-1} \Sigma_{0} \mathbf{e},
$$

where we have ignored the subscript ' + ' on $\tilde{Q}, \alpha_{L}$ and $\alpha_{R}$. If we choose $\alpha_{R}=-1$, the wave speed of the error is doubled in $\left[\epsilon_{1}, 1\right]$. By choosing $\alpha_{L}=2$, we get a reversed error wave speed of $-a$ in $\left[0, \epsilon_{0}\right]$.

In the experiments below we use the exact solution and initial data,

$$
u(x, t)=f(x-a t), \quad u(x, 0)=f(x)=e^{-100(x-0.5)^{2}} .
$$

We denote the error wave speed in $\left[\epsilon_{1}, 1\right]$ by $a_{R}$. The numerical solution moves with the wave speed $a$ in the whole domain $[0,1]$. However, in Figure 7 it can be seen that the error moves with $a_{R}=2 a$ in the region $\left[\epsilon_{1}, 1\right]$.

\subsection{The construction of non-reflecting boundary procedures}

Consider the problem (1.1) with zero boundary data and the initial condition $u(x, 0)=$ $e^{-600(x-0.5)^{2}}$. When the pulse leaves the domain at the outflow boundary, a portion of it 


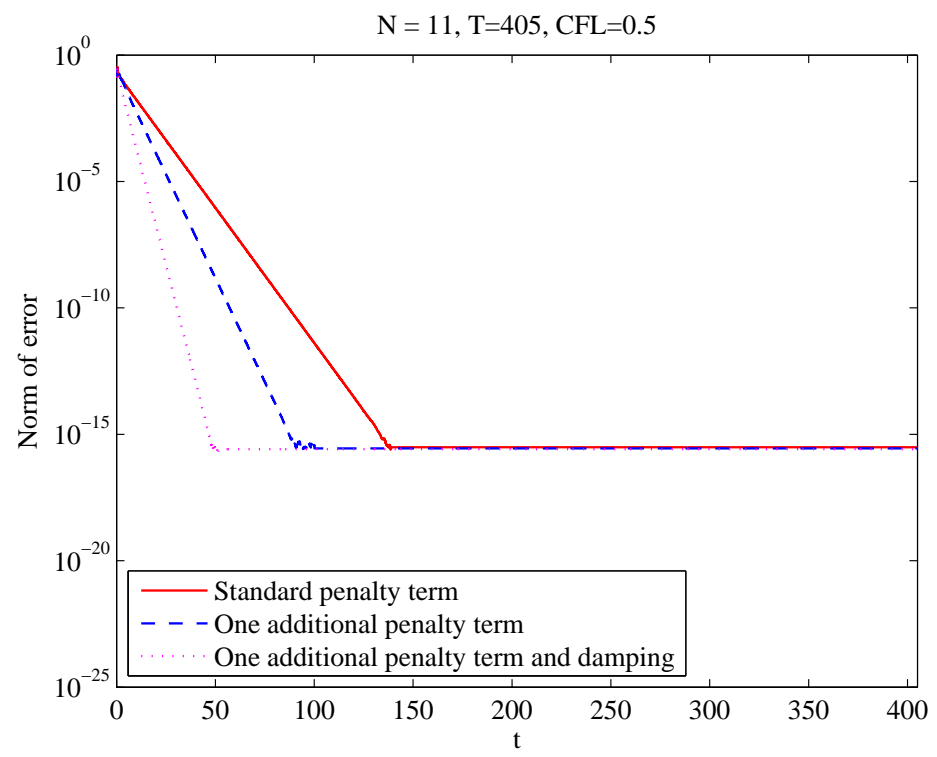

Figure 6: Convergence rate to steady-state using multiple penalty and additional damping on the outgoing waves given by 3.2 for $N=11$.
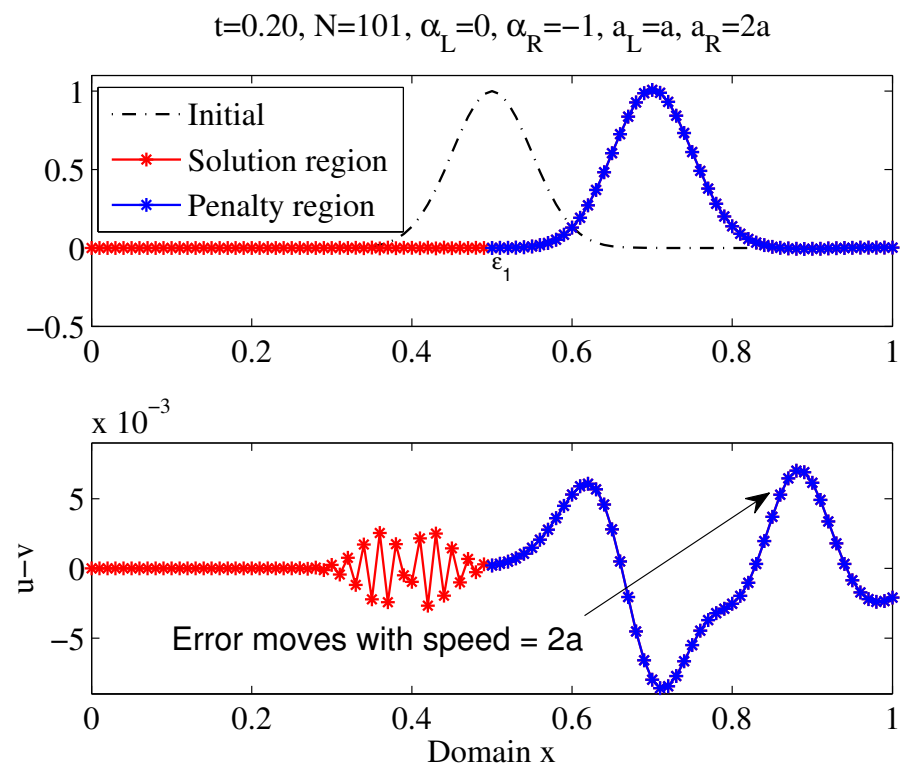

Figure 7: Error propagation with different speeds, $a_{L}=a, a_{R}=2 a$.

is reflected, creating an error in the computational domain. By considering a region $[\epsilon, 1]$ close to the outflow where multiple penalties can be applied, we can damp this error. The 


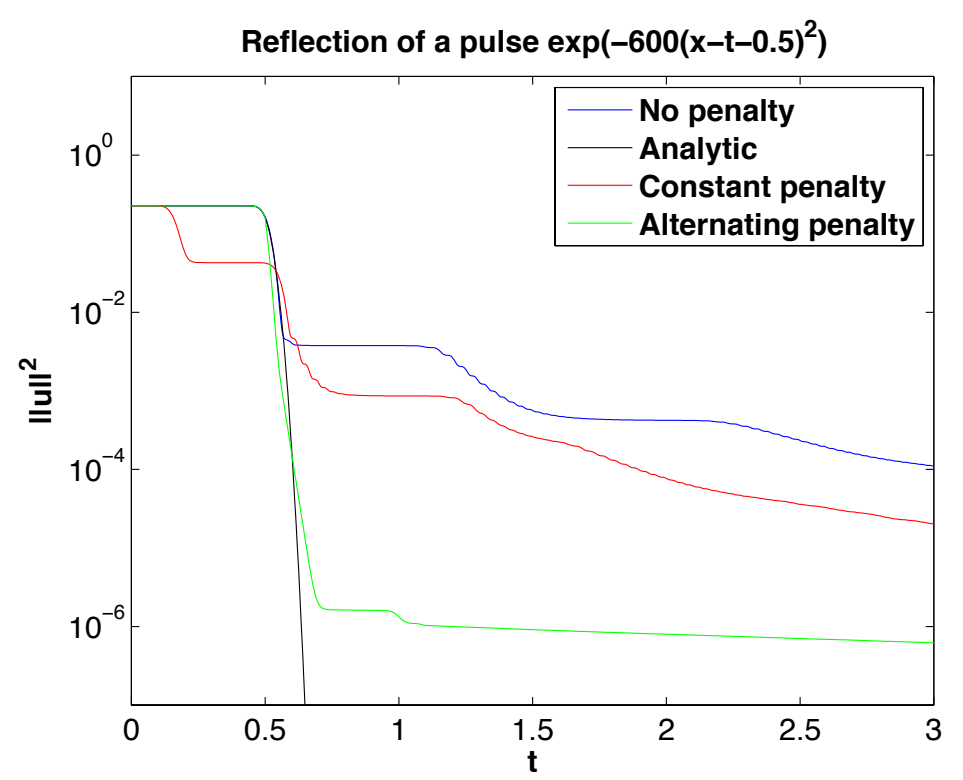

Figure 8: Error as a function of time when adding penalty terms for damping.

SBP-SAT method for this problem is then

$$
\mathbf{u}_{t}+a P^{-1} Q \mathbf{u}=-a P^{-1}\left(u_{0}-g_{0}\right) e_{0}-\frac{1}{2} \sum_{i \in[\epsilon, 1]} P^{-1}\left(u_{i}-g_{i}\right) e_{i}
$$

where we use $a=1$ and the same coefficient $(-1 / 2)$ for all the multiple penalty terms. In Figure 8, we can see that the reflected error is significantly decreased with multiple penalties in the region $[0.65,1]$. The additional reflections that occur at the interface between the computational and penalty domain are reduced by only applying the damping terms when the pulse has passed the interface and is close to the outflow boundary.

\subsection{Data dependence}

As the last part of the theory section we discuss how the multiple penalty technique depend on the accuracy and roughness of the data as well as the potential additional complication of non-linearity. We compare with standard requirements on boundary data.

With less accurate data the solution will loose accuracy, just as for inaccurate data for Dirichlet conditions. The only difference is that one needs accurate data at multiple points. The accuracy requirements are in principle the same as for standard weak Dirichlet boundary conditions.

Lack of smoothness in the data (the same as loss of accuracy if the underlying solution is smooth) can lead to oscillations in the numerical solution. However, most likely it is 
not a severe problem for this technique, since the data is imposed weakly. One way of reducing possible oscillations due to rough data would be to use penalty coefficients that are small in magnitude. This procedure however, requires using more terms to uphold the desired effect. We plan to investigate this problem/procedure in future work.

For non-linear problems, one needs to use non-linear penalty coefficients just as for standard penalty terms, see [15] for an example. It is important to make sure that the penalty terms are such that an energy estimate results. No other direct complication (except for the two already mentioned above) will occur.

In summary, the multiple penalty procedure is rather insensitive to bad data, and the requirements of accuracy and smoothness are no worse than on boundary data for standard boundary conditions. The main reason for the insensitivity is that the data is imposed weakly.

\section{Applications}

Here we will consider two different cases where the technique discussed above is used.

\subsection{Convergence to steady state of solutions to the Euler Equations}

We consider the Euler equations in one dimension. The governing equations are

$$
v_{t}+B v_{x}=0, \quad B=\left[\begin{array}{ccc}
u & \rho & 0 \\
0 & u & \frac{1}{\rho} \\
0 & \gamma & u
\end{array}\right]
$$

where $\gamma$ is the adiabatic index and $v=[\rho, u, p]^{T}$. The dependent variables $\rho, u$ and $p$ are the velocity, density and pressure respectively. We consider the final state of the convergence process and linearize (4.1) by considering a system close to an equilibrium state $v_{e q}=[\bar{\rho}, \bar{u}, \bar{p}]$. By exchanging $v=v-v_{e q}$ and neglecting all terms higher than first order in the Taylor series around $v_{e q}$, we obtain the linearized form of the Euler equations, with a coefficient matrix as given in (4.1) with $v$ replaced with $v_{e q}$. The frozen constant coefficient form of the system can be symmetrized, see [1,39], and the final result is

$$
u_{t}+A u_{x}=0, \quad A=\left[\begin{array}{ccc}
\bar{u} & \frac{1}{\sqrt{\gamma}} \bar{c} & 0 \\
\frac{1}{\sqrt{\gamma}} \bar{c} & \bar{u} & \sqrt{\frac{\gamma-1}{\gamma}} \bar{c} \\
0 & \sqrt{\frac{\gamma-1}{\gamma}} \bar{c} & \bar{u}
\end{array}\right], \quad u=\left[\begin{array}{c}
\frac{\bar{c} \rho}{\sqrt{\gamma} \bar{\rho}} \\
u \\
\frac{T}{\bar{c} \sqrt{\gamma(\gamma-1)}}
\end{array}\right] .
$$

We have introduced the speed of sound $c$ and also normalized the dependent variables using free stream values.

The final form of (4.2) describing the steady state process including boundary conditions becomes identical to (2.1). In our test cases, we consider subsonic flow with $\bar{c}=2 \bar{u}=2$ 


Table 4: Maximal real part of the discrete eigenvalues for standard and multiple penalties.
\begin{tabular}{|c|c|c|c|c|}
$\mathrm{N}$ & Standard penalty & +1 penalty & +2 penalties & +3 penalties \\
\hline 11 & -0.1642 & -0.1760 & -0.2828 & -0.4883 \\
21 & -0.0358 & -0.0374 & -0.0554 & -0.0838 \\
41 & -0.0084 & -0.0087 & -0.0124 & -0.0179
\end{tabular}

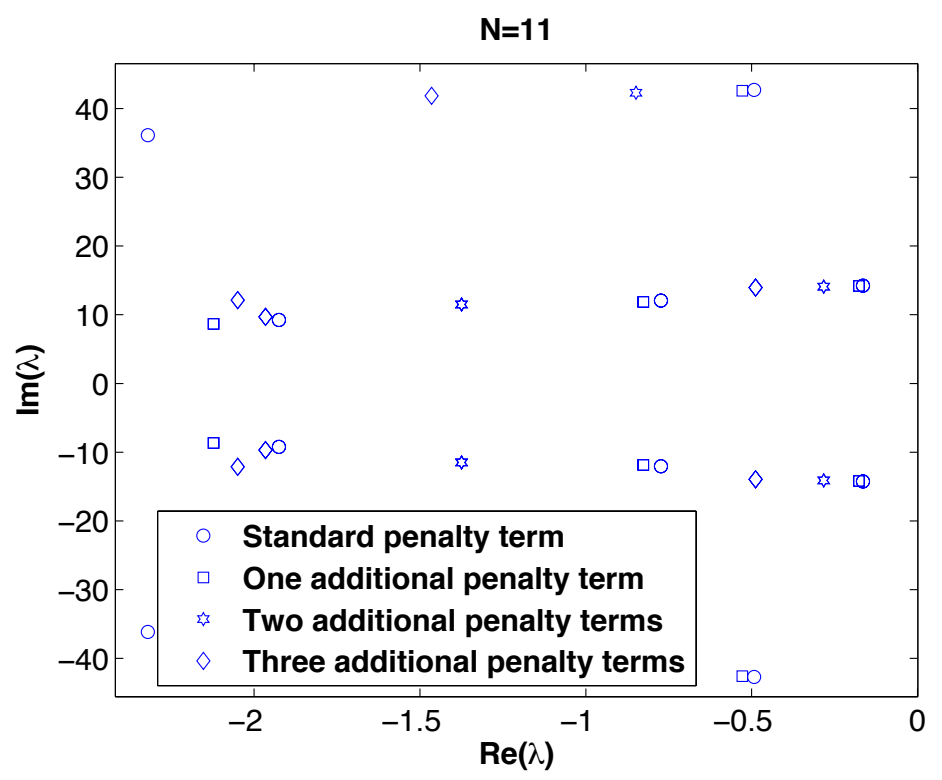

Figure 9: Effect on the spectrum when adding multiple penalties. $\mathrm{N}=11$. Note that the spectrum shifts left.

and $\gamma=1.4$. The additional multiple penalties are applied in both ends of the domain according to the technique described in section 2.2. For simplicity we have chosen the multiple penalty matrices as identity matrices multiplied by constants chosen such that the spectrum shifts away from the imaginary axis. The result on the spectrum is illustrated in Figure 9 and Table 4 . The SBP-SAT method applied to (2.1), (4.2) yields

$$
\begin{array}{r}
\mathbf{u}_{t}+P^{-1} Q \otimes A \mathbf{u}=-\left(P^{-1} E_{0} \otimes I\right)\left(E_{0} \otimes A^{+} u-g_{L}\right)+\left(P^{-1} E_{N} \otimes I\right)\left(E_{N} \otimes A^{-} u-g_{R}\right) \\
+\sum_{i} \alpha_{i}\left(P^{-1} E_{i} \otimes I\right)\left(E_{i} \otimes A^{+} u-g_{i}\right)+\alpha_{N-i}\left(P^{-1} E_{N-i} \otimes I\right)\left(E_{N-i} \otimes A^{-} u-g_{N-i}\right)
\end{array}
$$

where the matrices $E_{i}$ position the penalty terms at grid point $i$. The additional parameters $\alpha_{i} \leq 0, i \neq 0, N$ are chosen that such that we increase the rate of convergence. As an initial condition, we choose $u=1+e^{-100(x-0.5)^{2}}$ for all components of $u$. Computational results for $N=11,21$ are shown in Figure 10 and Figure 11 . The gain is considerable for $N=11$, but decreases for $N=21$. One can partly remedy that by adding additional terms as the mesh is refined. The results are consistent with Table 4 . 


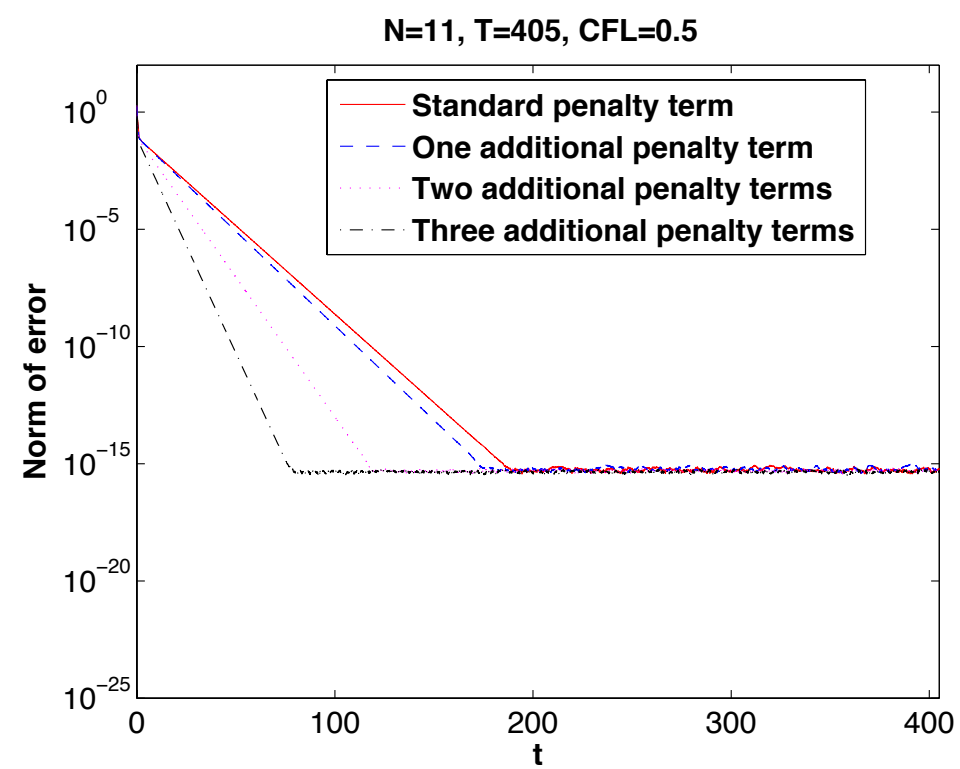

Figure 10: Convergence to steady-state of linearized Euler equations using multiple penalties. $N=11$

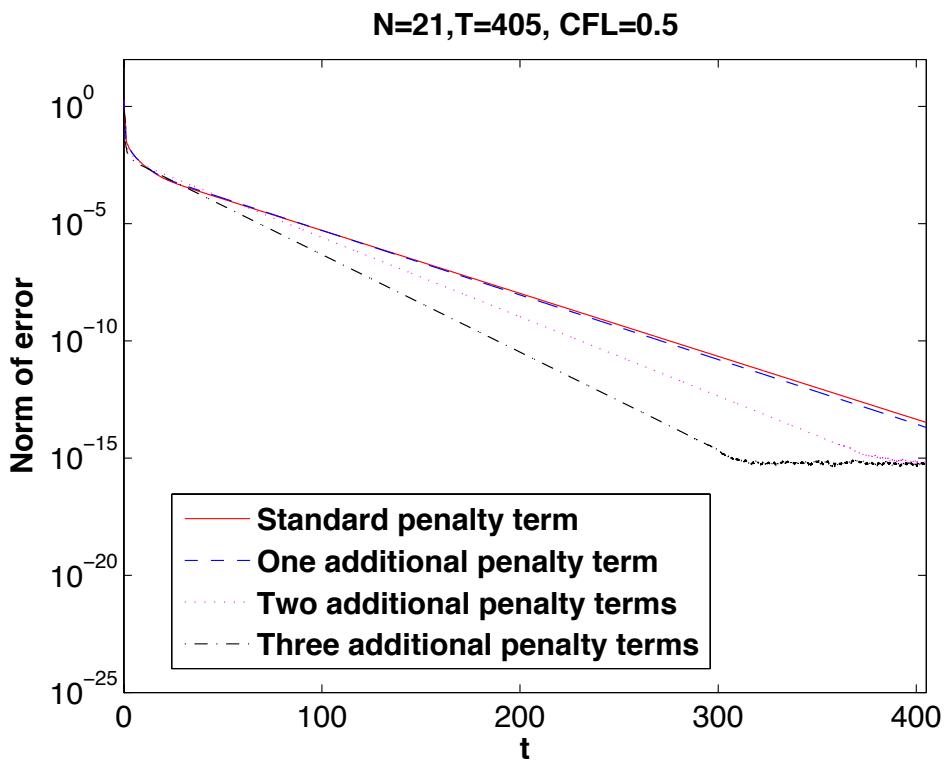

Figure 11: Convergence to steady-state of linearized Euler equations using multiple penalties. $N=21$ 


\subsection{A non-reflecting boundary procedure for the elastic wave equation}

In this section we consider a problem from geophysics, where a non-reflecting boundary procedure must be constructed. Assuming linear elasticity, and the validity of the elastic wave equations, the governing one-dimensional equations are

$$
\begin{array}{r}
\frac{\partial w}{\partial t}=B \frac{\partial w}{\partial y}, \quad B=\left[\begin{array}{cc}
0 & 1 / \rho \\
G & 0
\end{array}\right], \quad w=\left[\begin{array}{l}
v \\
\sigma
\end{array}\right], \quad y \in[0, H] \\
L_{o}(w)=\sigma(0, t)=F(V(t)), \quad L_{1}(w)=v(H, t)=v_{p} .
\end{array}
$$

The parameters $\rho$ and $G$ are the material density and shear modulus respectively. The boundary operators $L_{o}$ and $L_{1}$ act on the shear stress $\sigma$ and velocity $v$, respectively. We assume that a fault lies at $y=0$ and is governed by a boundary condition that equates shear stress with fault strength given through a friction law $F$ dependent on the particle velocity on the fault $V(t)=v(0, t)$. We set the velocity at the boundary $y=H$ to a slow "plate rate" $v_{p}$, intended to capture the effect of slow tectonic loading which will load the system and eventually cause a rupture to initiate at the fault.

\subsubsection{Analysis}

To analyze problem (4.4) we symmetrize the equations to

$$
\frac{\partial u}{\partial t}=A \frac{\partial u}{\partial y}, \quad A=\left[\begin{array}{cc}
0 & c_{s} \\
c_{s} & 0
\end{array}\right], \quad u=V^{-1} w=\left[\sqrt{\frac{\rho}{2}} v, \frac{1}{\sqrt{2 G}} \sigma\right]^{T},
$$

where $c_{s}=\sqrt{G / \rho}$ is the shear wave speed. Note that except for the specific form of the boundary conditions in (4.4 $\mathrm{b})$, the problem (4.5) is now of the form (2.1).

The non-conventional non-linear boundary condition in (4.4) forces a check of wellposedness, see [25, 26, 33]. The energy method applied to equation (4.5) yields

$$
\frac{d}{d t}\|u\|=2 \int_{0}^{H} u^{T} A u d y=\left.2 c_{s} u_{1} u_{2}\right|_{0} ^{H}=\left.v \sigma\right|_{0} ^{H}=-V F(V) \leq 0,
$$

with the assumption that $v_{p}=0$ and that the friction law $F$ has the physically relevant property that it takes the sign of its argument, i.e. $F(V) V \geq 0$. Uniqueness is obtained by considering the difference problem of the form (4.5) with identical data. It was shown in [33] that this requires $F^{\prime}(V) \geq 0$. By also observing that we give the right number of boundary conditions we can state that $(4.5)$ in combination with $(4.4 \mathrm{p})$ and hence (4.4) is well posed.

Next we assume that there exists an interval in the vicinity of $y=H$ where the system experiences loading at the plate rate. This means that we know that the velocity is equal to $v_{p}$ close to $y=H$ and we can therefore apply our multiple penalty technique in that domain without losing accuracy. 
Remark 4.1. Note that this situation is slightly different from the other situations in this paper where we have assumed that we know all variables of the solution in the penalty domain. Here we only know the value of one of the variables.

The semi-discrete form of the equations (4.4) is

$$
\left(P \otimes I_{2}\right) \mathbf{w}_{t}=(Q \otimes B) \mathbf{w}+\left(e_{0} \otimes \Sigma_{0}\left[\begin{array}{c}
\sigma_{0}-F\left(v_{0}\right) \\
\sigma_{0}-F\left(v_{0}\right)
\end{array}\right]\right)+\sum_{j=0}^{m-1}\left(e_{N-j} \otimes \Sigma_{N-j}\left[\begin{array}{c}
v_{N-j}-v_{p} \\
v_{N-j}-v_{p}
\end{array}\right]\right)
$$

where $\mathbf{w}=\left[v_{0} \sigma_{0} v_{1} \sigma_{1} \ldots v_{N} \sigma_{N}\right]^{T}$ is the vector of grid data, and $I_{2}$ is a $2 \times 2$ identity matrix. We symmetrize the matrix $B=V A V^{-1}$ as before. By letting $I_{N}$ denote the $N \times N$ identity matrix, equation (4.7) becomes

$$
\left(P \otimes I_{2}\right) \mathbf{u}_{t}=(Q \otimes A) \mathbf{u}+\left(e_{0} \otimes \tilde{\Sigma}_{0}\left[\begin{array}{c}
\sigma_{0}-F\left(v_{0}\right) \\
\sigma_{0}-F\left(v_{0}\right)
\end{array}\right]\right)+\sum_{j=0}^{m-1}\left(e_{N-j} \otimes \tilde{\Sigma}_{N-j}\left[\begin{array}{c}
v_{N-j}-v_{p} \\
v_{N-j}-v_{p}
\end{array}\right]\right)
$$

where $\tilde{\Sigma}_{0}=V^{-1} \Sigma_{0}, \tilde{\Sigma}_{N-j}=V^{-1} \Sigma_{N-j}$ and $u=\left(I_{N} \otimes V^{-1}\right) w$ is the scaled vector of grid data.

It can be shown, see [14], that the energy method applied to (4.8) and the symmetry properties of the SBP operators in combination with the penalty matrices

$$
\Sigma_{0}=\left[\begin{array}{cc}
\frac{1}{\rho} & 0 \\
0 & 0
\end{array}\right], \quad \Sigma_{N}=\left[\begin{array}{cc}
0 & 0 \\
0 & -G
\end{array}\right], \quad \Sigma_{N-j}=\left[\begin{array}{cc}
\beta_{j} & 0 \\
0 & 0
\end{array}\right], \quad \beta_{j} \leq 0, \quad j=1,2, \ldots, m-1
$$

lead to the estimate $\left(\|\mathbf{u}\|_{P \otimes I_{2}}^{2}\right)_{t} \leq-v_{0} F\left(v_{0}\right)$. Note that the discrete estimate is slightly more dissipative than the corresponding continuous one in (4.6). In summary, the approximation (4.7) of (4.4) in combination with (4.9) is stable. Next we will see how the additional semi-bounded parameters $\beta_{j}$ in 4.9 can be used.

\subsubsection{Numerical results}

The boundary condition at the fault $y=0$ is specified by a nonlinear friction law $F(V(t))$, see [11], [12], [13], [30] for more details. To discretize in time we use a backward-Euler adaptive-time stepping scheme [14] to numerically integrate equation (4.7) with penalty matrices given by (4.9). The initial conditions can all be specified by the initial slip velocity $V(0)$ which we take to be $V(0)=10^{-7} \mathrm{~m} / \mathrm{s} \approx 10 v_{p}$. For this simulation we take $v_{p}=32$ $\mathrm{mm} / \mathrm{a}, G=30 \mathrm{GPa}, c_{s}=3 \mathrm{~km} / \mathrm{s}, H=10 \mathrm{~km}$, and grid spacing $h=100 \mathrm{~m}$.

The system goes through an initial period of inter-seismic loading. As seen in Fig. 12 . a dynamic event initiates at the fault which sends out a wave through the domain, reflects off the boundary and returns to the fault. Once the wave exits the fault we use $m=200$ penalty terms close to $y=H$ (an interval of length $2 \mathrm{~km}$, which we refer to as the penalty domain) to impose the boundary condition $v(H, t)=v_{p}$ in order to suppress the wave as it exits the domain. The penalties are turned on once the pulse is contained within the penalty domain as seen in Fig. 13. We found that taking the constant value 


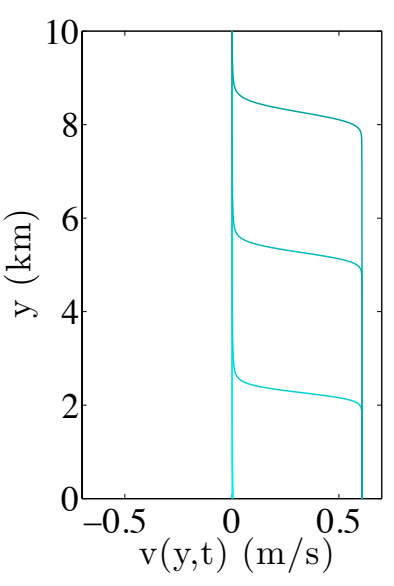

(a) $\mathrm{t}=0,1,2,3$ (s)

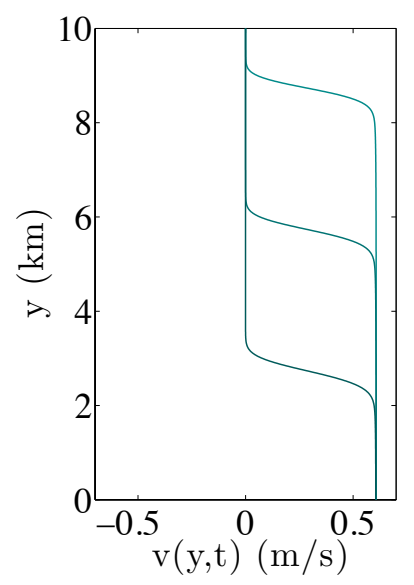

(b) $\mathrm{t}=4,5,6(\mathrm{~s})$

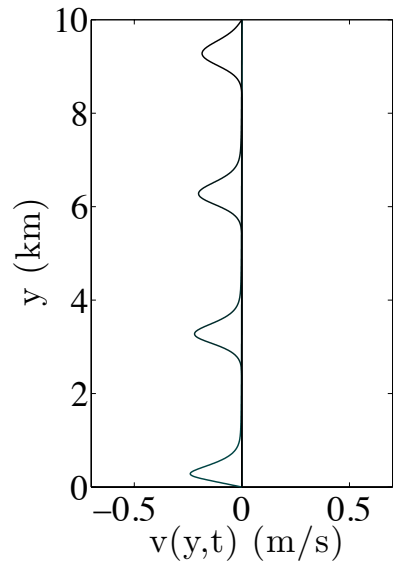

(c) $\mathrm{t}=7,8,9,10(\mathrm{~s})$

Figure 12: Snapshots of the particle velocity $v(y, t)$ plotted every 1 second during a dynamic event (time is after an approximate 30 year "interseismic" loading period). (a) The medium is essential at rest at $t=0$ (lightest blue vertical line). The wave is emitted at the fault $(y=0)$ and traverses the domain. It is plotted in progressively darker blue contours every second. (b) The wave is reflected from the boundary $y=H$ and back to the fault where it drives the velocity at the fault back to zero. (c) The wave returns to the fault and drives the slip velocity back to zero and travels back towards the remote boundary at $y=H$.

$\alpha_{j}=-600$ yields little reflections of the wave back into the domain, but further analysis needs to be done on how to choose their values. Without imposing the multiple penalty technique, the pulse continues to travel through the domain indefinitely.

\section{Conclusions}

We have introduced a new weak boundary procedure by applying penalty terms in extended domains. The technique can be used if one knows the exact solution in parts of the domain. The additional penalty terms do not add truncation error to the approximation and are added in a stable way.

After stability has been guaranteed, a number of free additional parameters are available. The additional parameters have been used to raise the the order of accuracy of the approximation, increase the rate of convergence to steady-state and to design nonreflecting boundary procedures.

The new boundary procedure is cheap, easy to implement, simple to program and in principle suitable for all numerical methods (not only finite difference methods) that employ weak boundary conditions.

We have exemplified the theoretical findings with numerical experiments, and the computational results are consistent with the theory. The extension of this method to more general problems involving dissipative terms will be investigated in future work. 


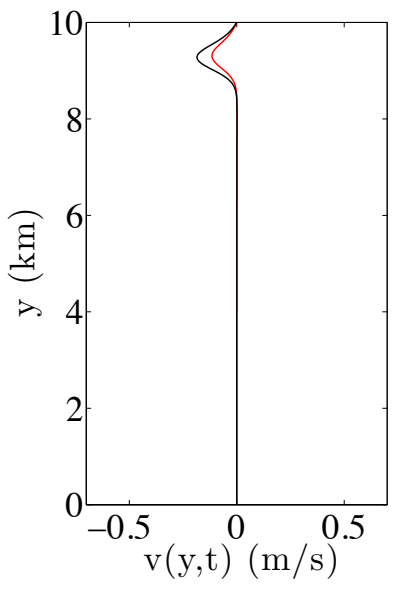

(a) $\mathrm{t}=10(\mathrm{~s})$

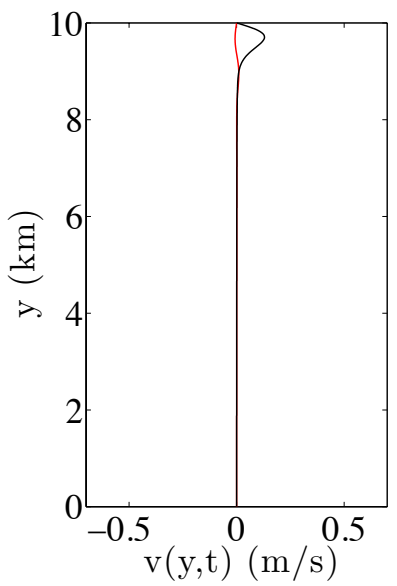

(d) $\mathrm{t}=10.3(\mathrm{~s})$

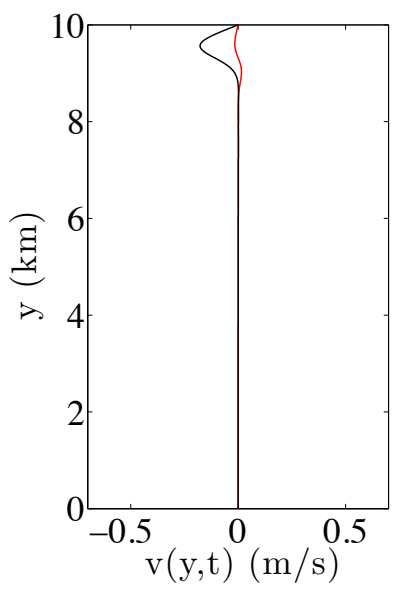

(b) $\mathrm{t}=10.1(\mathrm{~s})$

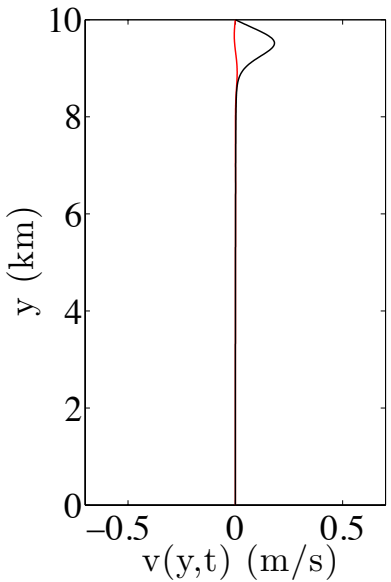

(e) $\mathrm{t}=10.4(\mathrm{~s})$

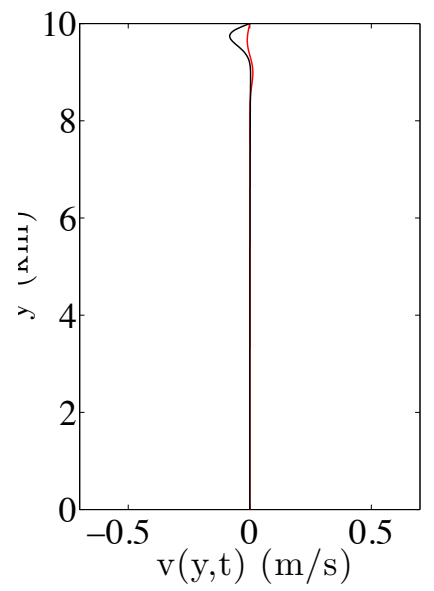

(c) $\mathrm{t}=10.2(\mathrm{~s})$

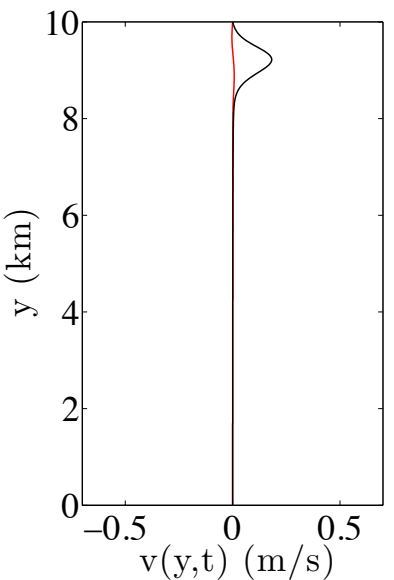

(f) $\mathrm{t}=10.5(\mathrm{~s})$

Figure 13: After the wave reaches the vicinity of $y=H$, the penalty terms are turned on. (a)-(f) show snapshots of particle velocity $v(y, t)$ plotted every tenth of a second. In black is the non-penalized solution, which should be compared to the penalized solution in red. At $t=10(\mathrm{~s})$ the penalties are turned on and the pulse decays over a time period of less than one second (about the time it takes for the non-penalized pulse to be reflected from the upper boundary). Without imposing the multiple penalty technique, the pulse continues to travel through the domain as seen in black. 


\section{Acknowledgements}

The work by the second author was supported by the Higher Education Commission (HEC) of Pakistan, while the second author was in residence at Uppsala University. Work by the third author was supported by the National Science Foundation under Award No. 0948304 and by the Southern California Earthquake Center. SCEC is funded by NSF Cooperative Agreement EAR-0529922 and USGS Cooperative Agreement 07HQAG0008 (SCEC contribution number 1806). The work by the last author was carried out within the Swedish e-science Research Centre (SeRC) and supported by the Swedish Research Council (VR).

\section{A Uniformly high order operators based on SBP norms}

For a uniformly second order accurate difference operator $P^{-1} Q$, the matrix $Q$ and the second order SBP norm $P$ are given as follows

$$
Q=\left[\begin{array}{ccccccc}
-\frac{3}{4} & 1 & -\frac{1}{4} & \cdots & & & 0 \\
-\frac{1}{2} & 0 & \frac{1}{2} & & & & \\
\vdots & & & \ddots & & & \vdots \\
& & & & -\frac{1}{2} & 0 & \frac{1}{2} \\
0 & & & \cdots & \frac{1}{4} & -1 & \frac{3}{4}
\end{array}\right], \quad P=\Delta x\left[\begin{array}{ccccc}
\frac{1}{2} & & & & 0 \\
& 1 & & & \\
& & \ddots & & \\
& & & 1 & \\
& & & & \frac{1}{2}
\end{array}\right]
$$

The matrix $\tilde{B}_{s}$ which raises the accuracy and the penalty matrix $\Sigma^{a c c}$ which maintain stability by making $\tilde{\Sigma}^{a c c}=0$ are given by

$$
\tilde{B}_{S}=\left[\begin{array}{cccccc}
-\frac{1}{2} & \frac{1}{2} & -\frac{1}{4} & \cdots & & 0 \\
\frac{1}{2} & 0 & & & & \\
-\frac{1}{4} & & & & & \\
\vdots & & & \ddots & & \vdots \\
& & & & \frac{1}{4} \\
& & & & 0 & -\frac{1}{2} \\
0 & & \cdots & \frac{1}{4} & -\frac{1}{2} & \frac{1}{2}
\end{array}\right], \quad \Sigma^{a c c}=\left[\begin{array}{ccc}
-\frac{1}{4} & & 0 \\
\frac{1}{2} & & \\
-\frac{1}{4} & & \\
\vdots & \ddots & \vdots \\
& & \frac{1}{4} \\
& & -\frac{1}{2} \\
0 & & \frac{1}{4}
\end{array}\right] .
$$


For a uniformly fourth order accurate difference operator on all grid points, we have

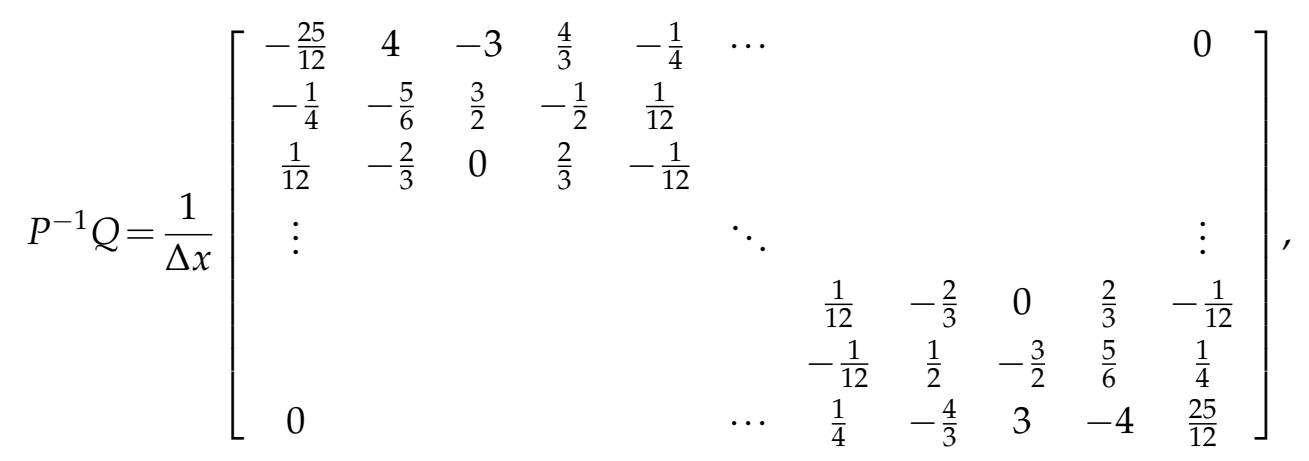

$$
P=\Delta x \operatorname{diag}\left(\begin{array}{lllllllllll}
\frac{17}{48} & \frac{59}{48} & \frac{43}{48} & \frac{49}{48} & 1 & \ldots & 1 & \frac{49}{48} & \frac{43}{48} & \frac{59}{48} & \frac{17}{48}
\end{array}\right)
$$

where $P$ is the SBP norm. We obtain $Q$ as

$$
Q=\left[\begin{array}{ccccccccccc}
-\frac{425}{576} & \frac{68}{48} & -\frac{51}{48} & \frac{17}{36} & -\frac{17}{192} & \cdots & & & & & 0 \\
-\frac{59}{192} & -\frac{295}{288} & \frac{59}{32} & -\frac{59}{96} & \frac{59}{576} & & & & & & \\
\frac{43}{576} & -\frac{43}{72} & 0 & \frac{43}{72} & -\frac{43}{576} & & & & & & \\
0 & \frac{49}{576} & -\frac{49}{72} & 0 & \frac{49}{72} & -\frac{49}{576} & & & & & \\
0 & 0 & \frac{1}{12} & -\frac{2}{3} & 0 & \frac{2}{3} & -\frac{1}{12} & & & & \\
\vdots & & & & & \ddots & & & & & \vdots \\
& & & & \frac{1}{12} & -\frac{2}{3} & 0 & \frac{2}{3} & -\frac{1}{12} & 0 & 0 \\
& & & & & \frac{49}{576} & -\frac{49}{72} & 0 & \frac{49}{72} & -\frac{49}{576} & 0 \\
& & & & & & \frac{43}{576} & -\frac{43}{72} & 0 & \frac{43}{72} & -\frac{43}{576} \\
0 & & & & & & -\frac{59}{576} & \frac{59}{96} & -\frac{59}{32} & \frac{295}{288} & \frac{59}{192} \\
& & & & \ldots & \frac{17}{192} & -\frac{17}{36} & \frac{51}{48} & -\frac{68}{48} & \frac{425}{576}
\end{array}\right]
$$


The matrix $\tilde{B}_{s}$ which raises the accuracy is

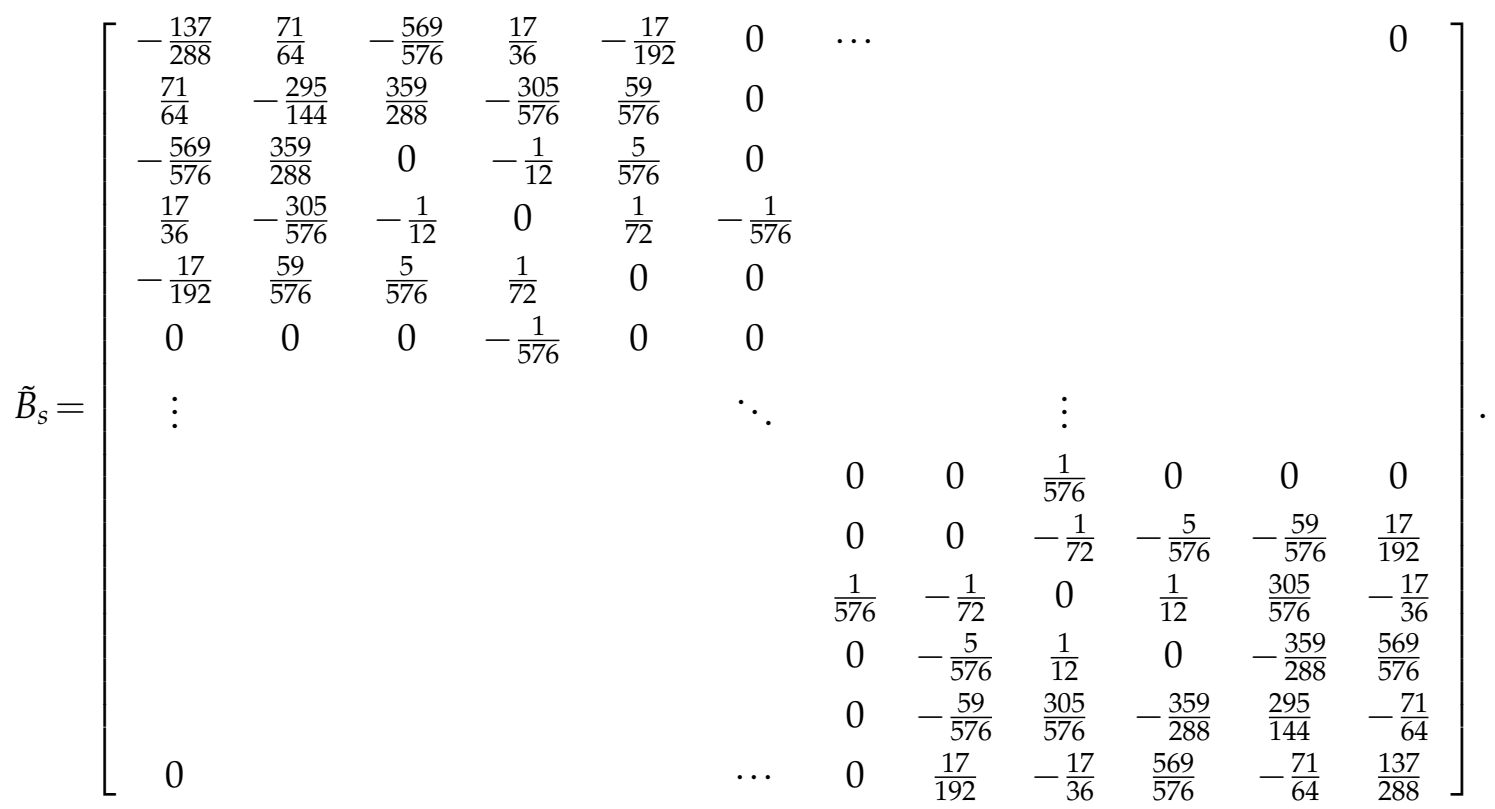

The penalty matrix $\Sigma^{a c c}$ which maintain stability by making $\tilde{\Sigma}^{a c c}=0$ is given by

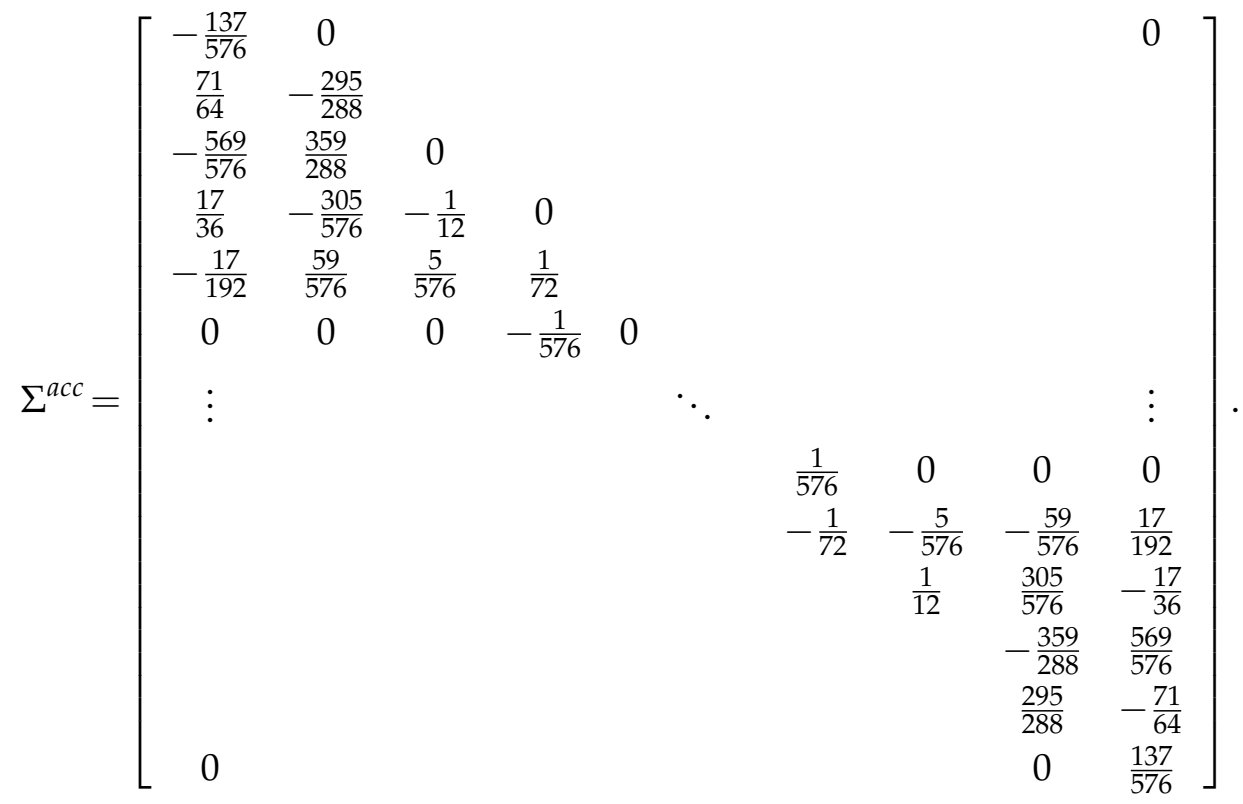


For a uniformly sixth order accurate difference operator on all grid points, we have

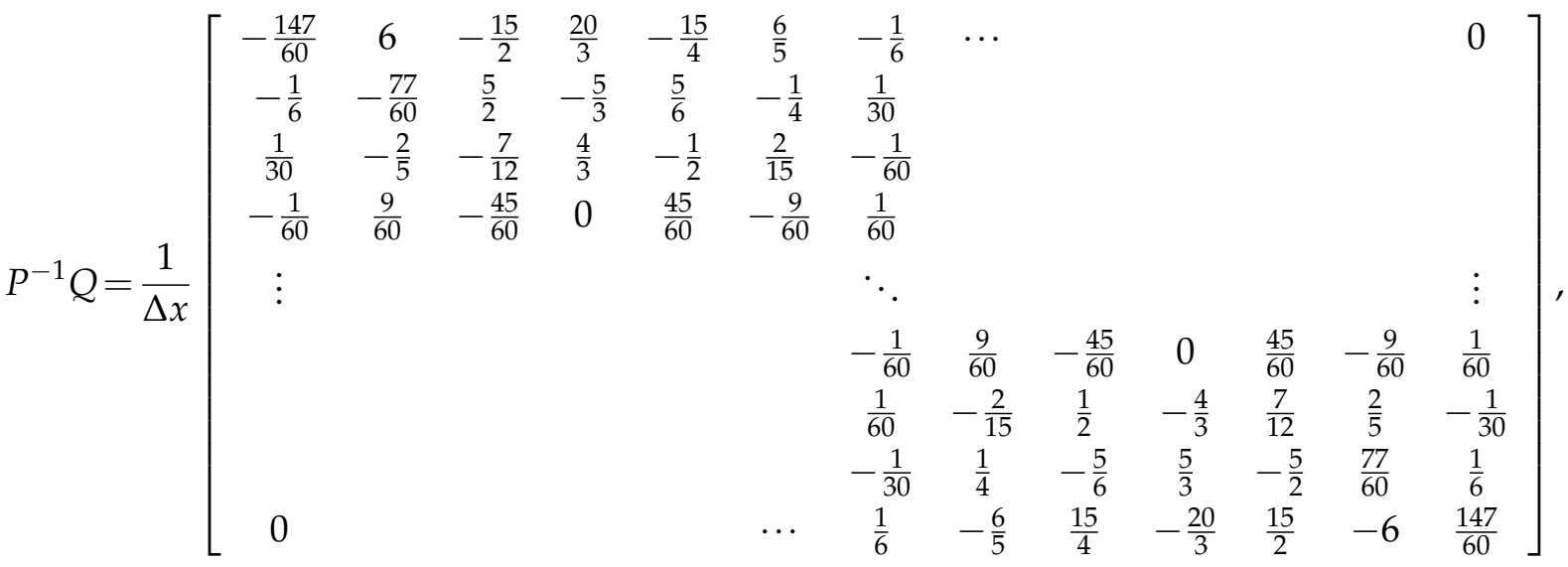

$$
\begin{aligned}
& P=\Delta x \operatorname{diag}\left(\begin{array}{cccccccc}
\frac{13649}{43200} & \frac{12013}{8640} & \frac{2711}{4320} & \frac{5359}{4320} & \frac{7877}{8640} & \frac{43801}{43200} & 1 & \ldots \\
& 1 & \frac{43801}{43200} & \frac{7877}{8640} & \frac{5359}{4320} & \frac{2711}{4320} & \frac{12013}{8640} & \frac{13649}{43200}
\end{array}\right)
\end{aligned}
$$

where $P$ is the corresponding SBP norm. We can get $Q$ at left boundary as

$$
Q=\left[\begin{array}{cccccccccc}
-\frac{668801}{864000} & \frac{13649}{7200} & -\frac{13649}{5760} & \frac{13649}{6480} & -\frac{13649}{11520} & \frac{13649}{36000} & -\frac{13649}{259200} & 0 & 0 & \cdots \\
-\frac{12013}{51840} & -\frac{925001}{518400} & \frac{12013}{3456} & -\frac{12013}{5184} & \frac{12013}{10368} & -\frac{12013}{34560} & \frac{12013}{259200} & 0 & 0 \\
\frac{2711}{129600} & -\frac{2711}{10800} & -\frac{18977}{51840} & \frac{2711}{3240} & -\frac{2711}{8640} & \frac{2711}{32400} & -\frac{2711}{259200} & 0 & 0 \\
-\frac{5359}{259200} & \frac{5359}{28800} & -\frac{5359}{5760} & 0 & \frac{5359}{5760} & -\frac{5359}{28800} & \frac{5359}{259200} & 0 & 0 \\
0 & -\frac{7877}{518400} & \frac{7877}{57600} & -\frac{7877}{11520} & 0 & \frac{7877}{11520} & -\frac{7877}{57600} & \frac{7877}{518400} & 0 & \\
0 & 0 & -\frac{43801}{5292000} & \frac{43801}{288000} & -\frac{43801}{57600} & 0 & \frac{43801}{57600} & -\frac{43801}{288000} & \frac{43801}{5292000} & \\
0 & 0 & 0 & -\frac{1}{60} & \frac{3}{20} & -\frac{3}{4} & 0 & \frac{3}{4} & \frac{3}{20} & \frac{1}{60} \\
\vdots & & & & & & & \ddots & & \vdots
\end{array}\right] .
$$

The matrix $\tilde{B}_{S}$ which raises the accuracy at left boundary is

$$
\tilde{B}_{S}=\left[\begin{array}{ccccccccc}
-\frac{236801}{432000} & \frac{431299}{259200} & -\frac{608783}{259200} & \frac{540601}{259200} & -\frac{13649}{11520} & \frac{13649}{36000} & -\frac{13649}{259200} & \ldots & \\
\frac{431299}{259200} & -\frac{925001}{259200} & \frac{92879}{28800} & -\frac{552419}{259200} & \frac{197591}{172800} & -\frac{12013}{34560} & \frac{12013}{259200} & & \\
-\frac{608783}{259200} & \frac{92879}{28800} & -\frac{18977}{25920} & -\frac{971}{10368} & -\frac{30589}{172800} & \frac{19231}{288000} & -\frac{2711}{259200} & & \\
\frac{540601}{259200} & -\frac{552419}{259200} & -\frac{971}{10368} & 0 & \frac{947}{3840} & -\frac{3263}{96000} & \frac{1039}{259200} & & \\
-\frac{13649}{11520} & \frac{197591}{172800} & -\frac{30589}{172800} & \frac{947}{3840} & 0 & -\frac{23}{300} & \frac{763}{57600} & -\frac{463}{314573} & \\
\frac{13649}{36000} & -\frac{12013}{34560} & \frac{19231}{288000} & -\frac{3263}{96000} & -\frac{23}{300} & 0 & \frac{601}{57600} & -\frac{601}{288000} & \frac{117}{504599} \\
-\frac{13649}{259200} & \frac{12013}{259200} & -\frac{2711}{259200} & \frac{1039}{259200} & \frac{763}{57600} & \frac{601}{57600} & 0 & & \\
0 & 0 & 0 & 0 & -\frac{463}{314573} & -\frac{601}{288000} & 0 & 0 & \\
0 & 0 & 0 & 0 & 0 & \frac{117}{504599} & 0 & 0 & 0 \\
\vdots
\end{array}\right.
$$


The penalty matrix $\Sigma^{a c c}$ which maintain stability by making $\tilde{\Sigma}^{a c c}=0$ at left boundary is given by

$$
\Sigma^{a c c}=\left[\begin{array}{cccccccccc}
-\frac{236801}{864000} & & & & & & & & & \\
\frac{431299}{259200} & -\frac{925001}{518400} & & & & & & & \\
-\frac{608783}{259200} & \frac{92879}{28800} & -\frac{18977}{51440} & & & & & & \\
\frac{540601}{259200} & -\frac{552419}{259200} & -\frac{971}{10368} & 0 & & & & & \\
-\frac{13649}{11520} & \frac{197591}{172800} & -\frac{30589}{172800} & \frac{947}{3840} & 0 & & & & \\
\frac{13649}{36000} & -\frac{12013}{34560} & \frac{19231}{288000} & -\frac{3263}{96000} & -\frac{23}{300} & 0 & & & \\
-\frac{13649}{259200} & \frac{12013}{259200} & -\frac{2711}{259200} & \frac{1039}{259200} & \frac{763}{57600} & \frac{601}{57600} & 0 & & \\
0 & 0 & 0 & 0 & -\frac{463}{314573} & -\frac{601}{288000} & 0 & 0 & & \\
0 & 0 & 0 & 0 & 0 & \frac{117}{504599} & 0 & 0 & 0 & \\
\vdots & & & & & & \ddots & & & \vdots
\end{array}\right]
$$

\section{B Uniformly high order operators based on identity norm}

For a uniformly second order accurate difference operator on all grid points, we use an identity norm $P=\Delta x I$ and obtain

$$
Q=\left[\begin{array}{ccccccc}
-\frac{3}{2} & 2 & -\frac{1}{2} & \cdots & & & 0 \\
-\frac{1}{2} & 0 & \frac{1}{2} & & & & \\
\vdots & & & \ddots & & & \vdots \\
& & & & -\frac{1}{2} & 0 & \frac{1}{2} \\
0 & & & \cdots & \frac{1}{2} & -2 & \frac{3}{2}
\end{array}\right]
$$

the matrix $\tilde{B}_{s}$ becomes

$$
\tilde{B}_{S}=\left[\begin{array}{cccccc}
-2 & \frac{3}{2} & -\frac{1}{2} & \cdots & & 0 \\
\frac{3}{2} & 0 & & & & \\
-\frac{1}{2} & & & & & \\
\vdots & & & \ddots & & \vdots \\
& & & & \frac{1}{2} \\
& & & & 0 & -\frac{3}{2} \\
0 & & \cdots & \frac{1}{2} & -\frac{3}{2} & 2
\end{array}\right] .
$$


The penalty matrix $\Sigma^{a c c}$ such that $\tilde{\Sigma}^{a c c}=0$ is given by

$$
\Sigma^{a c c}=\left[\begin{array}{ccc}
-1 & & 0 \\
\frac{3}{2} & & \\
-\frac{1}{2} & & \\
\vdots & \ddots & \vdots \\
& & \frac{1}{2} \\
& & -\frac{3}{2} \\
0 & & 1
\end{array}\right]
$$

For a uniformly fourth order accurate difference operator based on identity norm $P$, we have

$$
Q=\left[\begin{array}{ccccccccccc}
-\frac{25}{12} & 4 & -3 & \frac{4}{3} & -\frac{1}{4} & \cdots & & & & & 0 \\
-\frac{1}{4} & -\frac{5}{6} & \frac{3}{2} & -\frac{1}{2} & \frac{1}{12} & & & & & & \\
\frac{1}{12} & -\frac{2}{3} & 0 & \frac{2}{3} & -\frac{1}{12} & & & & & & \\
\vdots & & & & & \ddots & & & & & \vdots \\
& & & & & & \frac{1}{12} & -\frac{2}{3} & 0 & \frac{2}{3} & -\frac{1}{12} \\
& & & & & & -\frac{1}{12} & \frac{1}{2} & -\frac{3}{2} & \frac{5}{6} & \frac{1}{4} \\
0 & & & & & \ldots & \frac{1}{4} & -\frac{4}{3} & 3 & -4 & \frac{25}{12}
\end{array}\right]
$$

the matrix $\tilde{B}_{s}$ becomes

$$
\tilde{B}_{S}=\left[\begin{array}{ccccccc}
-\frac{19}{6} & \frac{15}{4} & -\frac{35}{12} & \frac{4}{3} & -\frac{1}{4} & \cdots & 0 \\
\frac{15}{4} & -\frac{5}{3} & \frac{5}{6} & -\frac{5}{12} & \frac{1}{12} & & \\
-\frac{35}{12} & \frac{5}{6} & & & & & \\
\frac{4}{3} & -\frac{5}{12} & & & & & \\
-\frac{1}{4} & \frac{1}{12} & & & & & \\
\vdots & & & \ddots & & & \vdots \\
& & & & & -\frac{1}{12} & \frac{1}{4} \\
& & & & & \frac{5}{12} & -\frac{4}{3} \\
& & & & & -\frac{5}{6} & \frac{35}{12} \\
& & -\frac{1}{12} & \frac{5}{12} & -\frac{5}{6} & \frac{5}{3} & -\frac{15}{4} \\
0 & \cdots & \frac{1}{4} & -\frac{4}{3} & \frac{35}{12} & -\frac{15}{4} & \frac{19}{6}
\end{array}\right] .
$$


The penalty matrix $\Sigma^{a c c}$ such that $\tilde{\Sigma}^{a c c}=0$ is given by

$$
\Sigma^{a c c}=\left[\begin{array}{cc}
-\frac{19}{12} & 0 \\
\frac{15}{4} & -\frac{5}{6} \\
-\frac{35}{12} & \frac{5}{6} \\
\frac{4}{3} & -\frac{5}{12} \\
-\frac{1}{4} & \frac{1}{12} \\
\vdots & \\
&
\end{array}\right.
$$$$
\left.\begin{array}{cc} 
& 0 \\
& \\
& \\
& \\
& \vdots \\
-\frac{1}{12} & \frac{1}{4} \\
\frac{5}{12} & -\frac{4}{3} \\
-\frac{5}{6} & \frac{35}{12} \\
\frac{5}{6} & -\frac{15}{4} \\
0 & \frac{19}{12}
\end{array}\right]
$$

For a uniformly sixth order accurate difference operator based on identity norm $P$, we have

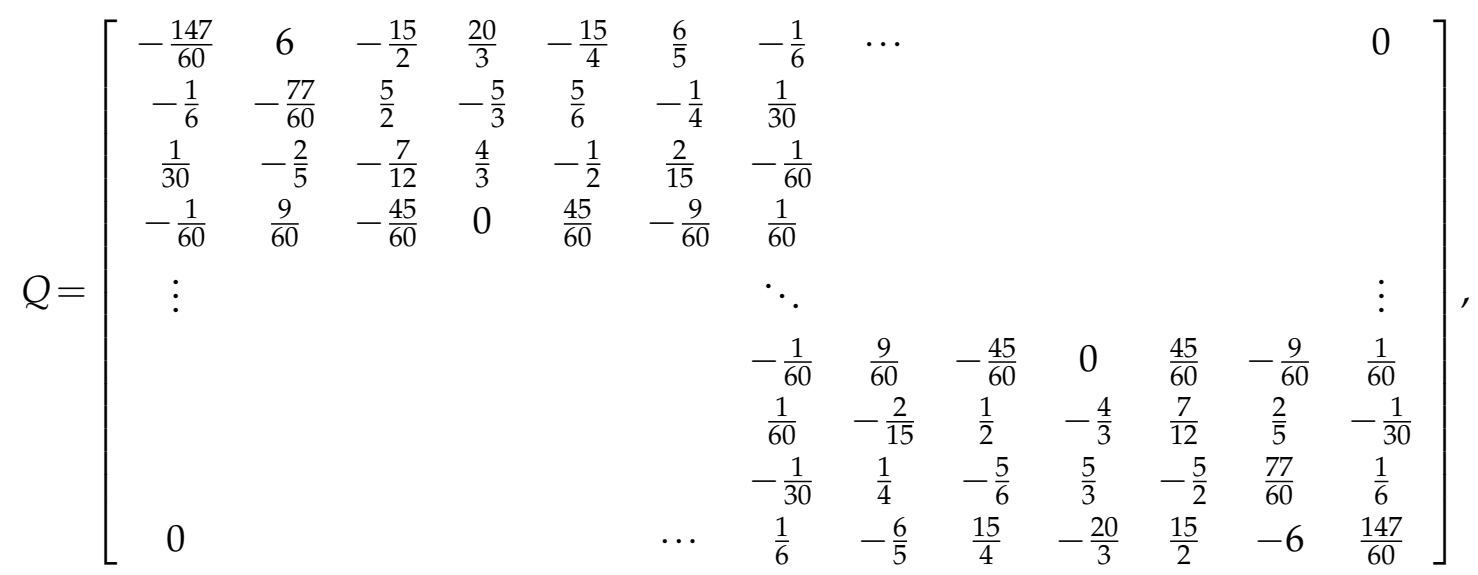


the matrix $\tilde{B}_{S}$ becomes

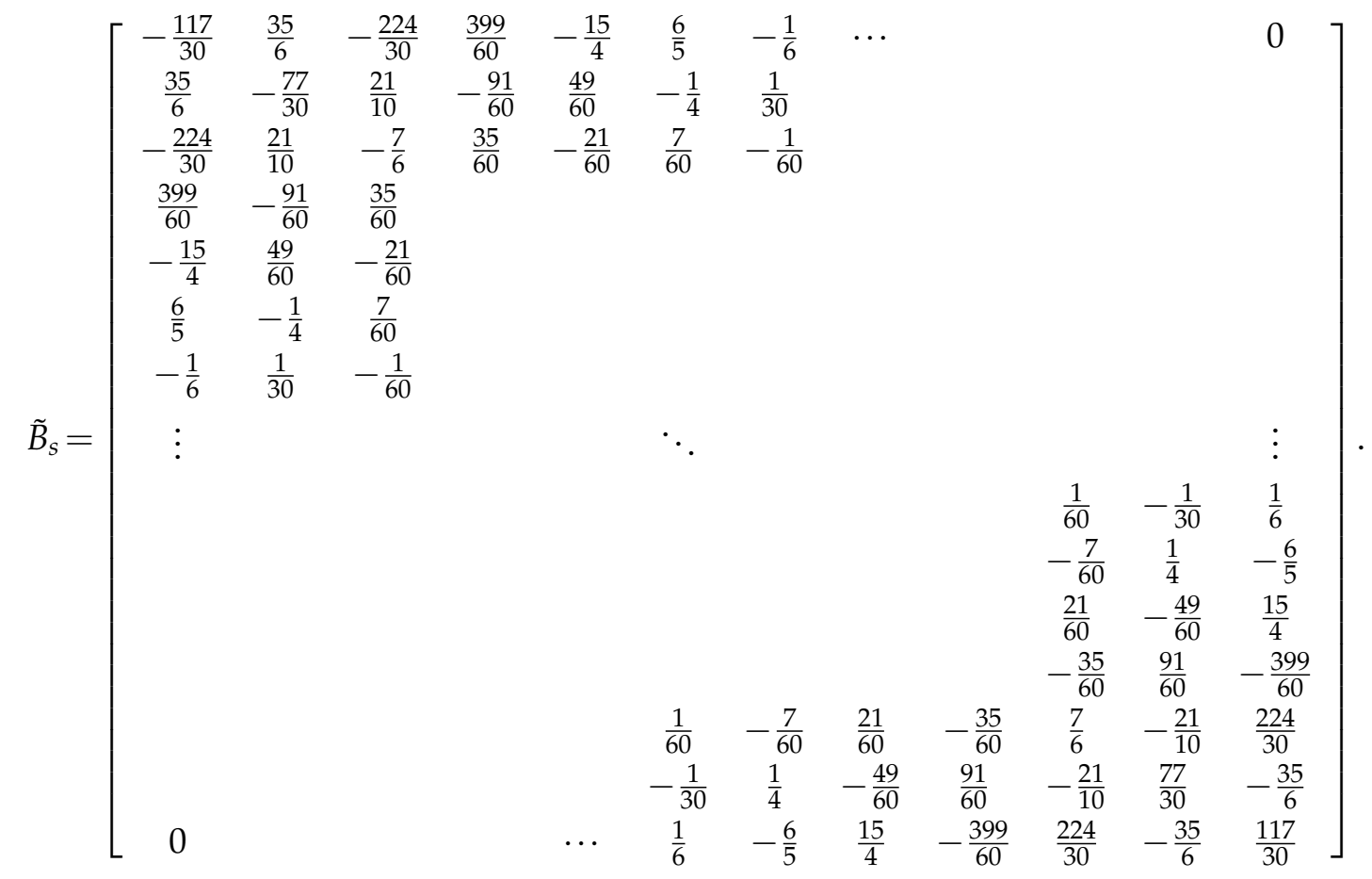

The penalty matrix $\Sigma^{a c c}$ such that $\tilde{\Sigma}^{a c c}=0$ is given by

$$
\Sigma^{a c c}=\left[\begin{array}{cccccccc}
-\frac{117}{60} & 0 & 0 & \cdots & & & & 0 \\
\frac{35}{6} & -\frac{77}{60} & 0 & & & & & \\
-\frac{224}{30} & \frac{21}{10} & -\frac{7}{12} & & & & \\
\frac{399}{60} & -\frac{91}{60} & \frac{35}{60} & & & & \\
-\frac{15}{4} & \frac{49}{60} & -\frac{21}{60} & & & & & \\
\frac{6}{5} & -\frac{1}{4} & \frac{7}{60} & & & & & \\
-\frac{1}{6} & \frac{1}{30} & -\frac{1}{60} & & & & & \\
\vdots & & & \ddots & & & & \vdots \\
& & & & & \frac{1}{60} & -\frac{1}{30} & \frac{1}{6} \\
& & & & & -\frac{7}{60} & \frac{1}{4} & -\frac{6}{5} \\
& & & & & \frac{21}{60} & -\frac{49}{60} & \frac{15}{4} \\
& & & & & -\frac{35}{60} & \frac{91}{60} & -\frac{399}{60} \\
& & & & & \frac{7}{12} & -\frac{21}{10} & \frac{224}{30} \\
& & & & & 0 & \frac{77}{60} & -\frac{35}{6} \\
0 & & & & \ldots & 0 & 0 & \frac{117}{60}
\end{array}\right]
$$




\section{Numerical boundary conditions}

Consider the problem (1.1), where the boundary condition is given at one point $x_{0}$,

$$
u_{0}=u\left(x_{0}, t\right)=g\left(x_{0}, t\right) .
$$

We generate boundary data numerically at grid points $x_{1}=x_{0}+h$ and $x_{2}=x_{0}+2 h$ by Taylor expansions

$$
\begin{aligned}
& u_{1}=u\left(x_{1}, t\right)=u_{0}+\left(u_{x}\right)_{0} h+\left(u_{x x}\right)_{0} \frac{h^{2}}{2 !}+\ldots+O\left(h^{p+1}\right), \\
& u_{2}=u\left(x_{2}, t\right)=u_{0}+\left(u_{x}\right)_{0}(2 h)+\left(u_{x x}\right)_{0} \frac{(2 h)^{2}}{2 !}+\ldots+O\left(h^{p+1}\right) .
\end{aligned}
$$

Here $p$ is the order of the scheme being used in computation. We can now compute all higher space derivatives by repeated differentiation as exemplified below

$$
\begin{aligned}
\left(u_{x}\right)_{0} & =-\left(u_{t}\right)_{0}=-g^{\prime}, \\
\left(u_{x x}\right)_{0} & =-\left(u_{x t}\right)_{0}=\left(u_{t t}\right)_{0}=g^{\prime \prime}, \\
\left(u_{x x x}\right)_{0} & =\left(u_{t t x}\right)_{0}=\left(u_{x t t}\right)_{0}=-\left(u_{t t t}\right)_{0}=-g^{\prime \prime \prime},
\end{aligned}
$$

where ' denotes the derivative with respect to $t$. Finally by using (C.3), we can obtain data to $u_{1}$ and $u_{2}$ by (C.2) to the required accuracy.

\section{References}

[1] S. Abarbanel and D. Gottlieb. Optimal time splitting for two- and three-dimensional NavierStokes equations with mixed derivatives. Journal of Computational Physics, 41(1):1-33, 1981.

[2] Q. Abbas and J. Nordström. Weak versus strong no-slip boundary conditions for the NavierStokes equations. Engineering Applications of Computational Fluid Mechanics, 4(1):29-38, 2010.

[3] J. Berg and J. Nordström. Stable robin solid wall boundary conditions for the Navier-Stokes equations. Journal of Computational Physics, 230(19):7519-7532, 2011.

[4] D. J. Bodony. Analysis of sponge zones for computational fluid mechanics. Journal of Computational Physics, 212(2):681-702, 2006.

[5] D. J. Bodony. Accuracy of the simultaneous-approximation-term boundary condition for time-dependent problems. Journal of Scientific Computing, 43(1):118-133, 2010.

[6] M. H. Carpenter, J. Nordström, and D. Gottlieb. Revisiting and extending interface penalties for multi-domain summation-by-parts operators. Journal of Scientific Computing, 45(1-3):118150, 2010.

[7] M.H. Carpenter, D. Gottlieb, and S. Abarbanel. Time-stable boundary conditions for finitedifference schemes solving hyperbolic systems: methodology and application to high-order compact schemes. Journal of Computational Physics, 111(2):220-236, 1994.

[8] M.H. Carpenter, J. Nordström, and D. Gottlieb. A stable and conservative interface treatment of arbitrary spatial accuracy. Journal of Computational Physics, 148:341-365, 1999.

[9] T. Colonius. Modeling artificial boundary conditions for compressible flow. Annual Review of Fluid Mechanics, 336:315-345, 2004. 
[10] H.C. Davies. A lateral boundary formulation for multilevel prediction models. Quart. J.R. Met. Soc., 102:405-418, 1976.

[11] J. Dieterich. Time-dependent friction and the mechanics of stick-slip. Pure appl. Geophys., 116:790-806, 1978.

[12] J. H Dieterich. Modeling of rock friction, 1, experimental results and constitutive equations. J. Geophy. Res., 84:2161-2168, 1979a.

[13] J. H. Dieterich and B. D. Kilgore. Direct observation of frictional contacts: new insights for state dependent properties. Pure Appl. Geophys., 143:283-302, 1994.

[14] B. Erickson and J. Nordström. Stable, high order accurate adaptive schemes for long time highly intermittent geophysics problems. In prep., 2013.

[15] Travis C. Fisher, Mark H. Carpenter, Jan Nordström, Nail K. Yamaleev, and Charles Swanson. Discretely conservative finite-difference formulations for nonlinear conservation laws in split form: Theory and boundary conditions. Journal of Computational Physics, 234:353-375, 2013.

[16] J. Gong and J. Nordström. Interface procedures for finite difference approximations of the advectiondiffusion equation. Journal of Computational and Applied Mathematics, 236(5):602620, 2011.

[17] B. Gustafsson. Far-field boundary conditions for time-dependent hyperbolic systems. SIAM Journal on Scientific and Statistical Computing, 9(3):812-828, 1988.

[18] B. Gustafsson and H. O. Kreiss. Boundary conditions for time dependent problems with an artificial boundary. Journal of Computational Physics, 30(3):333-351, 1979.

[19] B. Gustafsson, H.O. Kreiss, and J. Oliger. Time dependent Problems and Difference Methods. Wiley-Interscience, New York, 1995.

[20] Bertil Gustafsson. High Order Difference Methods for Time Dependent PDE. Number 38 in Springer Series in Computational Mathematics. Springer-Verlag, 2008.

[21] T. Hagstrom. Radiation boundary conditions for the numerical simulation of waves. Acta Numerica, 8:47-106, 1999.

[22] J. E. Hicken and D. W. Zingg. Parallel Newton-Krylov solver for the Euler equations discretized using simultaneous-approximation terms. AIAA Journal, 46(11):2773-2786, 2008.

[23] X. Huan, J. E. Hicken, and D. W. Zingg. Interface and boundary schemes for high-order methods. In The 39th AIAA Fluid Dynamics Conference, San Antonio, USA, 22-25 June 2009, June 2009. AIAA Paper No. 2009-3658.

[24] P. Kållberg. Test of a lateral boundary relaxation scheme in a barotropic model. Report 3, European Centre for Medium Range Weather Forecasts, Bracknell, United Kingdom, 1977.

[25] J. E. Kozdon, E. M. Dunham, and J. Nordström. Interaction of waves with frictional interfaces using summation-by-parts difference operators: Weak enforcement of nonlinear boundary conditions. J. Sci. Comput., 50:341-367, 2012.

[26] J. E. Kozdon, E. M. Dunham, and J. Nordstrm. Simulation of dynamic earthquake ruptures in complex geometries using high-order finite difference methods. Journal of Scientific Computing, 55(1):92-124, 2013.

[27] H.O. Kreiss and J. Oliger. Comparison of accurate methods for the integration of hyperbolic equations. Tellus, 24:199-215, 1972.

[28] H.O. Kreiss and G. Scherer. Finite element and finite difference methods for hyperbolic partial differential equations, Mathematical Aspects of Finite Elements in Partial Differential Equations. Academic Press, Inc., 1974.

[29] H.O. Kreiss and G. Scherer. On the existence of energy estimates for difference approximations for hyperbolic systems. Technical report, Department of Scientific Computing, Uppsala 
University, 1977.

[30] C. Marone. Laboratory-derived friction laws and their application to seismic faulting. Annu. Rev. Earth Planet. Sci., 26:643-696, 1998.

[31] K. Mattsson. Boundary procedures for summation-by-parts operators. Journal of Scientific Computing, 18(1):133-153, 2003.

[32] J. Nordström. Conservative finite difference formulations, variable coefficients, energy estimates and artificial dissipation. 29(3):375-404, 2006.

[33] J. Nordström. Linear and nonlinear boundary conditions for wave propagation problems, volume 120 of Notes on Numerical Fluid Mechanics and Multidisciplinary Design. 2013.

[34] J. Nordström and M.H. Carpenter. Boundary and interface conditions for high-order finitedifference methods applied to the Euler and Navier-Stokes equations. Journal of Computational Physics, 148(2):621-645, 1999.

[35] J. Nordström and M.H. Carpenter. High-order finite difference methods, multidimensional linear problems, and curvilinear coordinates. Journal of Computational Physics, 173(1):149$174,2001$.

[36] J. Nordström and S. Eriksson. Fluid structure interaction problems: The necessity of a well posed, stable and accurate formulation. Communications in Computational Physics, 8(5):1111$1138,2010$.

[37] J. Nordström, J. Gong, E. van der Weide, and M. Svärd. A stable and conservative high order multi-block method for the compressible Navier-Stokes equations. Journal of Computational Physics, 228(24):9020-9035, 2009.

[38] J. Nordström, N. Nordin, and D. Henningson. The fringe region technique and the fourier method used in the direct numerical simulation of spatially evolving viscous flows. SIAM Journal of Scientific Computing, 20(4):1365-1393, 1999.

[39] J. Nordström and M. Svärd. Well posed boundary conditions for the Navier-Stokes equations. SIAM Journal of Numerical Analysis, 43(3):1231-1255, 2005.

[40] P. Olsson. Summation by parts, projections, and stability, i. Mathematics of Computation, 64(211):1035-1065, 1995.

[41] P. Olsson. Summation by parts, projections, and stability, ii. Mathematics of Computation, 64(212):1473-1493, 1995.

[42] Bo Strand. Summation by parts for finite difference approximations for $d / d x$. Journal of Computational Physics, 110(1):47-67, 1994.

[43] M. Svärd. On coordinate transformations for summation-by-parts operators. Journal of Scientific Computing, 20(1):29-42, 2004.

[44] M. Svärd, M.H. Carpenter, and J. Nordström. A stable high-order finite difference scheme for the compressible Navier-Stokes equations: far-field boundary conditions. Journal of Computational Physics, 225(1):1020-1038, 2007.

[45] M. Svärd and J. Nordström. On the order of accuracy for difference approximations of initial-boundary value problems. Journal of Computational Physics, 218(1):333-352, 2006.

[46] M. Svärd and J. Nordström. A stable high-order finite difference scheme for the compressible Navier-Stokes equations: no-slip wall boundary conditions. Journal of Computational Physics, 227(10):4805-4824, 2007.

[47] S. V. Tsynkov. Numerical solution of problems on unbounded domains. a review. Applied Numerical Mathematics, 27(4):465-532, 1998.

[48] D. W. Zingg. Comparison of high-accuracy finite-difference methods for linear wave propagation. SIAM Journal on Scientific Computing, 22(2):476-502, 2001. 